\title{
Comparative whole genome DNA methylation profiling across cattle tissues reveals global and tissue-specific methylation patterns
}

Yang Zhou ${ }^{1 *+}$, Shuli Liu ${ }^{2,3+}$, Yan Hu ${ }^{1+}$, Lingzhao Fang ${ }^{4}$, Yahui Gao ${ }^{2}$, Han Xia ${ }^{1}$, Steven G. Schroeder ${ }^{2}$, Benjamin D. Rosen², Erin E. Connor ${ }^{5}$, Cong-jun Li ${ }^{2}$ Ransom L. Baldwin², John B. Cole ${ }^{2}$, Curtis P. Van Tassell ${ }^{2}$, Liguo Yang ${ }^{1}$, Li Ma ${ }^{6}$ and George E. Liü ${ }^{2^{*}}$

\begin{abstract}
Background: Efforts to improve animal health, and understand genetic bases for production, may benefit from a comprehensive analysis of animal genomes and epigenomes. Although DNA methylation has been well studied in humans and other model species, its distribution patterns and regulatory impacts in cattle are still largely unknown. Here, we present the largest collection of cattle DNA methylation epigenomic data to date.

Results: Using Holstein cattle, we generated 29 whole genome bisulfite sequencing (WGBS) datasets for 16 tissues, 47 corresponding RNA-seq datasets, and 2 whole genome sequencing datasets. We did read mapping and DNA methylation calling based on two different cattle assemblies, demonstrating the high quality of the long-read-based assembly markedly improved DNA methylation results. We observed large differences across cattle tissues in the methylation patterns of global CpG sites, partially methylated domains (PMDs), hypomethylated regions (HMRs), CG islands (CGIs), and common repeats. We detected that each tissue had a distinct set of PMDs, which showed tissue-specific patterns. Similar to human PMD, cattle PMDs were often linked to a general decrease of gene expression and a decrease in active histone marks and related to longrange chromatin organizations, like topologically associated domains (TADs). We tested a classification of the HMRs based on their distributions relative to transcription start sites (TSSs) and detected tissue-specific TSS-HMRs and genes that showed strong tissue effects. When performing cross-species comparisons of paired genes (two opposite strand genes with their TSS located in the same HMR), we found out they were more consistently co-expressed among human, mouse, sheep, goat, yak, pig, and chicken, but showed lower consistent ratios in more divergent species. We further used these WGBS data to detect 50,023 experimentally supported CGls across bovine tissues and found that they might function as a guard against C-to-T mutations for TSS-HMRs. Although common repeats were often heavily methylated, some young Bov-A2 repeats were hypomethylated in sperm and could affect the promoter structures by exposing potential transcription factor binding sites.

(Continued on next page)
\end{abstract}

\footnotetext{
* Correspondence: yangzhou@mail.hzau.edu.cn; George.Liu@usda.gov

†Yang Zhou, Shuli Liu and Yan Hu contributed equally to this work.

'Key Laboratory of Agricultural Animal Genetics, Breeding and Reproduction

of Ministry of Education \& College of Animal Science and Technology,

Huazhong Agricultural University, Wuhan 430070, China

${ }^{2}$ Animal Genomics and Improvement Laboratory, Agricultural Research

Service, USDA, Beltsville, MD 20705, USA

Full list of author information is available at the end of the article
}

(C) The Author(s). 2020 Open Access This article is licensed under a Creative Commons Attribution 4.0 International License, which permits use, sharing, adaptation, distribution and reproduction in any medium or format, as long as you give appropriate credit to the original author(s) and the source, provide a link to the Creative Commons licence, and indicate if changes were made. The images or other third party material in this article are included in the article's Creative Commons licence, unless indicated otherwise in a credit line to the material. If material is not included in the article's Creative Commons licence and your intended use is not permitted by statutory regulation or exceeds the permitted use, you will need to obtain permission directly from the copyright holder. To view a copy of this licence, visit http://creativecommons.org/licenses/by/4.0/ The Creative Commons Public Domain Dedication waiver (http://creativecommons.org/publicdomain/zero/1.0/) applies to the data made available in this article, unless otherwise stated in a credit line to the data. 
(Continued from previous page)

Conclusions: This study provides a comprehensive resource for bovine epigenomic research and enables new discoveries about DNA methylation and its role in complex traits.

Keywords: Cattle, Somatic tissues, DNA methylation, Partially methylated domains, Hypomethylated region, WGBS (whole genome bisulfite sequencing)

\section{Background}

DNA methylation plays important roles in tissue differentiation and normal developmental processes like gene expression, genomic imprinting, repression of transposable elements, and gametogenesis [1-5]. Many tissuespecific differentially methylated regions (DMRs) were identified and proposed to mediate tissue-specific gene regulatory mechanisms in humans [6]. Earlier studies profiling DNA methylomes in humans and rodents have also shown low methylation near promoters and high methylation in the bodies of active genes [7, 8]. But the relationship between methylation and expression is context-dependent. For example, Varley et al. reported that CpG-rich enhancers in the bodies of expressed genes are actually unmethylated [9].

Partially methylated domains (PMDs) were first discovered in human cell lines and cancers [10]. PMDs were later detected in most mammalian placentas and mouse germline cells [11-14], covering up to $75 \%$ of the genomes. As one of the prominent signatures of longrange epigenomic organization, PMDs are large domains of DNA (often greater than $100 \mathrm{~kb}$ ) which have lower levels of DNA methylation and are associated with gene repression. Early human and mouse analysis identified PMDs as important general, lineage-, and cell typespecific topological features [15]. As Salhab et al. [15] pointed out, changes in PMDs are hallmarks of cell differentiation, with decreased methylation levels and increased heterochromatic histone marks, which are linked to domains of early, middle, and late DNA replication and cell proliferation. However, the patterns and the function impacts of PMDs in cattle are still not known.

CpG sites occur with high frequency in genomic regions called CpG islands (CGIs), which are one of the most widely studied regulatory features. Commonly used cattle CGIs are usually predicted from DNA sequence using computer programs, such as the one downloaded from the University of California Santa Cruz (UCSC) Genome Browser [16]. Although CGIs have critical roles in development and disease, recent studies have shown that such computational annotations are not totally accurate [17]. On the other hand, hypomethylated regions (HMRs, hundreds bp in length) often are located in CGIs and linked to the activation of gene expression; however, they also occur outside of CGIs and function as cell type-specific enhancers [9]. As has been reported [18-20], the formation of HMRs can be due to two possible mechanisms: (1) active transcription and accompanying histone marks such as H3K4me3 prevent the access of DNA methyltransferases and (2) specific protein/DNA complexes, such as CTCF and Sp1, inhibit the methylation machinery in the absence of transcription.

Compared to somatic cells, sperm cells undergo nearly complete reprogramming of DNA methylation and exchange histones by protamine [21-23]. We previously profiled the DNA methylome of cattle sperm through comparison with somatic cells from three bovine tissues (mammary gland, brain, and blood) [24]. Large differences between cattle sperm and somatic cells were observed in the methylation patterns of global CpGs, pericentromeric satellites, and common repeats. Although most of common repeats were heavily methylated in both sperm and somatic cells, we did find some hypomethylated repeats were enriched in gene promoters of sperm cells. Common repeats or transposable elements constitute roughly half of most mammalian genomes [25]. Repression of these common repeats relies on DNA methylation via the piRNA pathway and is essential for the maintenance of genomic stability in the long term and for germ cell function in the short term $[26,27]$. In humans, common repeats were found to be heavily methylated-with the notable exclusion of young AluY and AluYa5 elements in human sperm cells [28]. If methylation is lost on certain repressed repeats, germ cell development is arrested in meiosis [29].

Our knowledge of DNA methylation patterns in livestock is still limited when compared to humans and other model species. Some DNA methylation studies were reported with limited tissue types and/or low resolution in cattle, pigs, sheep, and horses [24, 30-46]. For example, we performed comparative analyses of sperm DNA methylomes among human, mouse, and cattle and provided insights into epigenomic evolution and complex traits [47]. To understand the variability of DNA methylation across cattle tissues and its regulation of gene expression, we profiled the cattle DNA methylomes in 16 major tissues using the whole genome bisulfite sequencing (WGBS) method. We investigated the landscapes of the DNA methylome across tissues. We studied differential methylation by comparing them in multiple contexts, including global CpG sites, PMDs, 
HMRs, CGIs, and common repeats. In line with the Functional Annotation of Animal Genome (FAANG) project [48], this study provides a comprehensive resource for bovine epigenomic research and enables new discoveries about DNA methylation and its role in complex traits.

\section{Results}

\section{Data generation and quality assessment}

We generated 29 WGBS datasets for 16 tissues from 2 Holstein cows and their relatives, including biological replicates whenever possible. These also included 47 corresponding transcriptome datasets for 14 of the 16 tissues and 2 whole genome sequencing datasets (Additional file 2: Table S1A and S1B). Besides 10 published datasets (4 sperm, 2 brain prefrontal cortex, 2 mammary gland, 2 whole blood samples from GSE106538, 24), the other 19 WGBS datasets were newly generated from samples of 2 rumen, 2 lung, 2 Latissimus dorsi muscle, 2 adipose, 1 heart, 1 ileum, 1 liver, 1 kidney, 1 spleen, 1 ovary, and 1 uterus collected from the two cows, as well as 2 white blood cell and 2 placental samples from their 4 female relatives. We obtained vast amounts of data, and for each of them, the average unique mapped read count was approximately 150 million (Additional file 2: Table S1). We then uniformly applied Bismark [49] for read mapping and DNA methylation calling, based on two cattle assemblies, i.e., short-read-based UMD3.1.1 [50] versus long-read-based ARS-UCD1.2 [51]. Although the differences of mapping rates and global methylation levels were small between two different assemblies (Additional file 2: Table S2), many DNA methylation-level peaks and valleys did change their locations and magnitudes, especially when they were near chromosome ends (i.e., pericentromeres or telomeres) or sudden drops of the DNA methylation level (Additional file 1: Figure S1 and S2). Considering the high quality of the long-read assembly, such as the better context identification and future applicability, we focused all following analyses using the long-read-based assembly ARS-UCD1.2. We used Bismark [49] to identify genome-wide methylated cytosines, which gave a median coverage of $16 \times$ (coverage) per sample (range from 11.84 to $24.47 \times$ ) (Additional file 2: Table S1). To remove the SNP artifacts from the subsequent analyses, we filtered away all SNPs detected in the whole genome sequence of the same individual (Additional file 2: Table S1C).

\section{Dynamic DNA methylation for tissue-specific development in cattle}

In terms of the global CpG methylation level of the cattle genome DNA, we obtained consistent results as compared to human [52], mouse [53], and other species. Most somatic tissue DNA samples in cattle had average methylation levels of $70 \sim 80 \%$, while the placental genomic DNA was $48 \%$ (the least) methylated, as compared to the sperm DNA which was $~ 78 \%$ (the most) methylated (Additional file 2: Table S1A). Bisulfite conversion rates estimated by unmethylated lambda DNA controls supported that we faithfully captured patterns of genomic DNA methylation in these samples (Additional file 2: Table S1A). Moreover, we detected low non-CG methylation in the non-brain somatic tissues and sperm cells $(0.2-0.8 \%)$, in contrast to a higher non-CG methylation level in the brain samples (1.2-1.3\%). The latter was consistent with previous studies in human and other species [54].

We performed a hierarchical clustering of these tissues based on weighted methylation levels of 500-bp windows (Fig. 1a). As expected, they were organized into three main clusters: sperm cells (cluster 1), placenta (cluster 2 ), and somatic tissues (cluster 3). These results confirmed the consistent results between the biological replicates and reinforced potential methylation differences among different somatic tissues, placentas, and sperm cells (Fig. 1a). Additionally, independent principal component analysis (PCA) also confirmed this clustering (Additional file 1: Figure S3a). PC1 successfully separated samples into 3 clusters (sperm, placentas, and somatic tissues), which explained most (44.90\%) of the variances, while PC2 separated sperm and placentas from somatic tissues. PC3 separated the blood/white blood cell/spleen from the rest and PC4 separated the rumen from all other samples.

We compared the methylation profiles between pairs of samples at a global CpG level. As expected, the correlations between samples within the same tissue type or clusters were high $(r>0.5)$ (Additional file 1: Figure S4). The correlations between methylation of different tissue types or clusters were lower, especially those between sperm cells and somatic tissues were the lowest $(r=0.22$ to 0.24) (Additional file 1: Figure S4).

Focusing on cluster 3 of Fig. 1a, we saw much more conserved methylation patterns when they were compared to the sperm cells and placenta in all 3 analyses (clustering, PCA, and correlation in Fig. 1a, S3, and S4, respectively). The somatic tissues in cluster 3 can be further divided into 3 branches with stronger correlations among their DNA methylome levels (Fig. 1a). It is noted that samples from the same germ layers and/or the same biological systems were clustered together (e.g., heart and muscle, spleen and blood, and adipose tissue and mammary gland). This was in agreement with the common notion about the importance of methylation in tissue-specific development. Moreover, several tissues showed specific methylation patterns in cattle. For example, the blood cluster (cluster b) and the rumen were separately divided by PC3 (6.54\%) and PC4 (4.39\%) from 
(a)

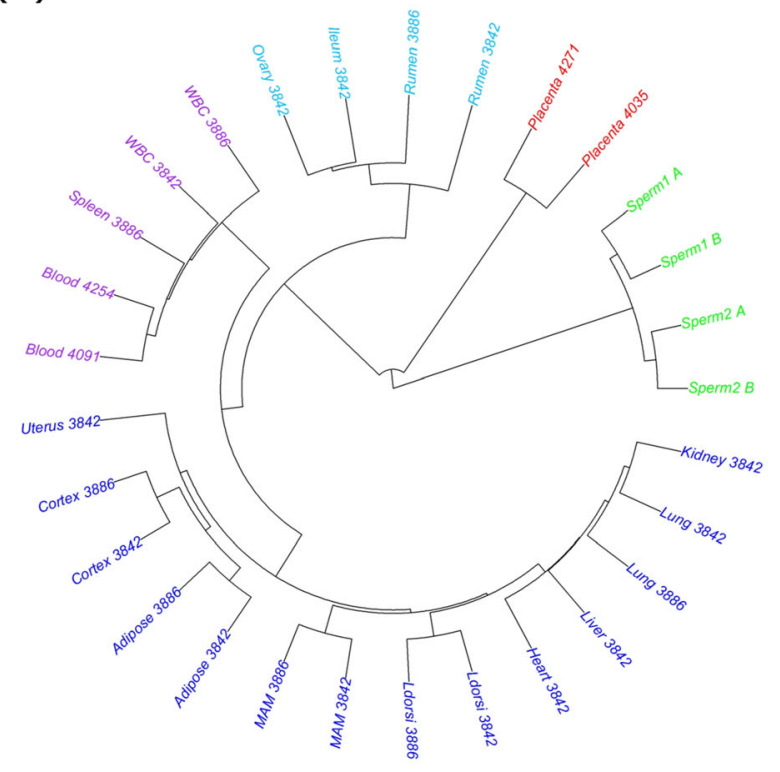

(b)

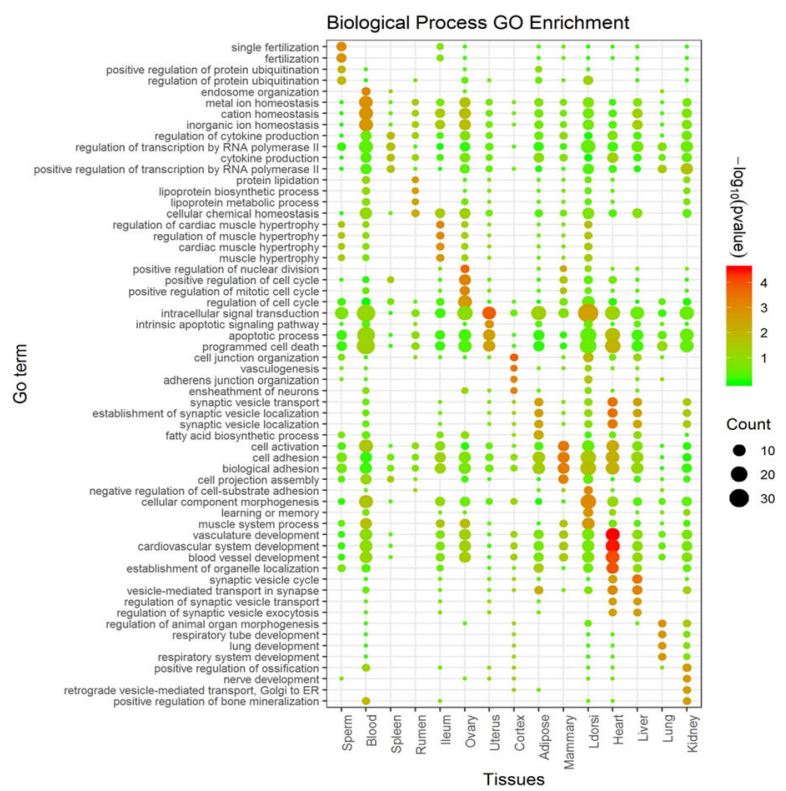

Fig. 1 Analyses of DNA methylation for the tissue-specific development in cattle. a Cluster analysis of the samples using the average methylation level of 500-bp windows. b Gene ontology analysis for the genes overlapped with tissue-specific DMRs

other tissues (Additional file 1: Figure S3b). Interestingly, we also found increased correlation coefficients of methylation levels toward the sperm cells and placenta especially for the cortex and rumen, respectively (Additional file 1: Figure S5). Using 10-kb, non-overlapping windows, we analyzed the conserved and variable methylation regions on the cattle genome across all samples, based on the standard deviations of DNA methylation levels. We defined the lowest $1 \%$ tails as methylation conserved regions, while the highest $1 \%$ tails as methylation variable regions (Additional file 1: Figure S6). We first divided methylation conserved regions into 2 parts: hypermethylation conserved regions and hypomethylation conserved regions. We found that genic regions were most enriched in hypermethylation conserved regions (Additional file 1: Figure S7). The genes located in the hypermethylation conserved regions were highly enriched in the DNA damage and repair biological processes (Additional file 1: Figure S8a). In the hypomethylation conserved regions, some of important functional genomic features including promoters, eCpG islands, and tRNA genes were highly enriched (Additional file 1: Figure S9). But no significant enriched GO term was found. On the other hand, we found that methylation variable regions were enriched in the promoters and eCGIs (Additional file 1: Figure S10). No significantly enriched GO term was found when we did the GO analysis using all the genes overlapped with the methylation variable regions (Additional file 1: Figure S11). We then separated methylation variable regions into 2 parts according to the sperm methylation level: sperm hypermethylation variable regions and sperm hypomethylation variable regions. We found that the genes located in the sperm hypomethylation variable regions (the methylation variable regions that showed hypomethylation in sperm) showed high enrichment in the meiotic cell cycle process, cell division, and spermatogenesis (Additional file 1: Figure S8b). The genes located in the sperm hypermethylation variable regions (the methylation variable regions that showed hypermethylation in sperm) showed high enrichment in the response to hormone, multi-multicellular organism process (Additional file 1: Figure S8c). Additionally, methylation variable regions in our heatmap analysis (Additional file 1: Figure S12) successfully separated the samples into three similar groups (placenta, sperm, and other somatic tissues), as we observed in our hierarchical clustering based on weighted methylation levels (top 500-bp tails) (Fig. 1a). Therefore, as described above, our results showed that the methylation conserved regions played important roles for the basic, key bioprocesses, while the methylation variable regions were associated with tissue-specific activities.

We identified 5.85 million differentially methylated cytosines (DMCs, methylation differences >0.3, FDR < 0.05 , with $10 \times$ in depth) that distributed in 215,984 differentially methylated regions (DMRs, methylation differences $>0.3$, FDR $<0.05$, supported by at least $5 \mathrm{DMCs}$ in the same direction) between any one tissue sample against tissues in all other groups throughout the cattle genome (Additional file 2: Table S3). As the placenta 
was lowly methylated, we detected the largest number of DMCs and DMRs covering half of the genome. Among somatic tissues and sperm cells, we identified the tissuespecific DMRs and found the hypomethylated genes overlapped with the tissue-specific DMRs were enriched in the tissue-specific development GO terms, for example, fertilization for the sperm, positive regulation of the nuclear division for the ovary, and vasculature development for the heart (Fig. 1b). These results indicated large differences between tissue methylomes were likely related to tissue-specific development and function.

\section{PMD}

As described before [24], we applied an HMM model to detect partially methylated domains (PMDs) using 10-kb windows (Additional file 2: Table S4), whose lengths are usually over hundreds of kilobases. Previous studies have recognized that the placenta has a similar epigenetic landscape as cancer cells have, which are characterized by a widespread hypomethylation in human and mice [13]. Here, we found that the cattle placenta contributed $80 \%(\sim 1.2 \mathrm{~Gb})$ of all PMDs in length and covered most of the PMDs detected in the other tissues. Cattle placenta PMDs covered 40.83 and $43.74 \%$ of the cattle genomes, respectively. On the other hand, PMD only occupied $30.29 \%$ of the human placenta genome, agreeing with previous estimates [13]. We further determined genes located in the shared and lineage-specific placenta PMDs between cattle and human (Additional file 2: Table S5). GO terms with significant enrichment for genes shared in PMDs included chemical synaptic transmission, adhesion junction organization, ion transmembrane transport, and nervous system development. Genes for human-specific PMDs showed one marginally significant enrichment for anterior/posterior pattern specification, while cattle-specific PMDs showed one significant enrichment for steroid hormone-mediated signaling pathway (Additional file 2: Table S6).

After merging all cattle tissue PMDs, we found that over half $(\sim 1.45 \mathrm{~Gb})$ of the whole cattle genome were covered by PMDs in at least one sample. However, PMD mostly existed in the gene desert (gene poor region) with low CG density and often lack of the actively histone modifications, including H3K27Ac and H3K4me3 (Fig. 2a, Additional file 2: Table S4). Overlapping with the $\mathrm{Hi}-\mathrm{C}$ contact maps revealed that TADs were often associated with PMDs on the cattle genome (Fig. 2b). We did find several PMDs that overlapped with parts of the genes or gene cluster regions. But most of PMDs often showed a genome-wide inhibition of gene expression in all samples (Additional file 1: Figure S13). Moreover, we identified highly methylation domains (HMDs) using the HMM strategy in the placenta. Placenta HMDs covered $27.58 \%$ of the cattle genome and were enriched for the gene-related features, including genic regions, promoters, experimentally supported CGIs (eCGIs, presented in the later part of the "Results" section) (Additional file 1: Figure S14), and 7057 (54.80\%) RefGenes. In PMDs, the methylation patterns of the genes and the CGI were almost indistinguishable from the background because of their low methylation (Additional file 1: Figure S15). But the genes in HMDs were significantly enriched in the basic biological processes, including intracellular protein transport, DNA repair, apoptotic process, endocytosis, and cell division (Additional file 2: Table S7). This may help to explain how the placenta functions normally even with a large percentage of PMDs. Genes in placenta PMDs were significantly enriched in the following GO terms: homophilic cell adhesion via plasma membrane adhesion molecules, gamma-aminobutyric acid signaling pathway, pituitary gland development, chemical synaptic transmission, and anterior/posterior pattern specification (Additional file 2: Table S8).

We further examined whether the cattle PMDs could be used as markers for different tissues. We found the replicates for the same tissues were successfully clustered together using the PMDs (Fig. 2c). Most of the samples, except the placenta, had small proportions of PMDs. Within them, we found the rumen, heart, liver, and ovary had more PMDs than others. It is of note that the blood and its related sample (spleen) showed the least numbers and shortest lengths of PMDs (Additional file 2: Table S4). Through a visual examination, we detected multiple methylation-level drops that commonly appeared in the non-blood samples at the chromosome level (Fig. 2a labeled with narrow squares, and Additional file 2: Table S9). We then identified those DNA methylation-level drop regions on the chromosome using a PMD-cluster strategy. Finally, we identified 16 non-blood DNA methylation-level drops ranging from $0.3 \sim 1.8 \mathrm{Mb}$ in length, which were distributed on 13 different chromosomes (Additional file 2: Table S9). Five of them overlapped with gene cluster regions with gene family loci related to immunity, histone, olfactory receptor, pregnancy-associated glycoprotein, and protocadherin. The others were enriched in satellite. Since all bovine autosomes are acrocentric and pericentromeres are not well defined in the cattle genome, the first $3 \mathrm{Mb}$ of them could be considered as potential pericentromeres. Based on this criterion, 9/16 of them were located in pericentromeres (Additional file 2: Table S9).

\section{TSS-HMR as an important indicator for gene expression}

We also applied an HMM module to detect the hypomethylated regions (HMRs) for each sample, as described previously [24]. In total, we found that the HMR covered $847 \mathrm{Mb}$ of the cattle genome. However, the 


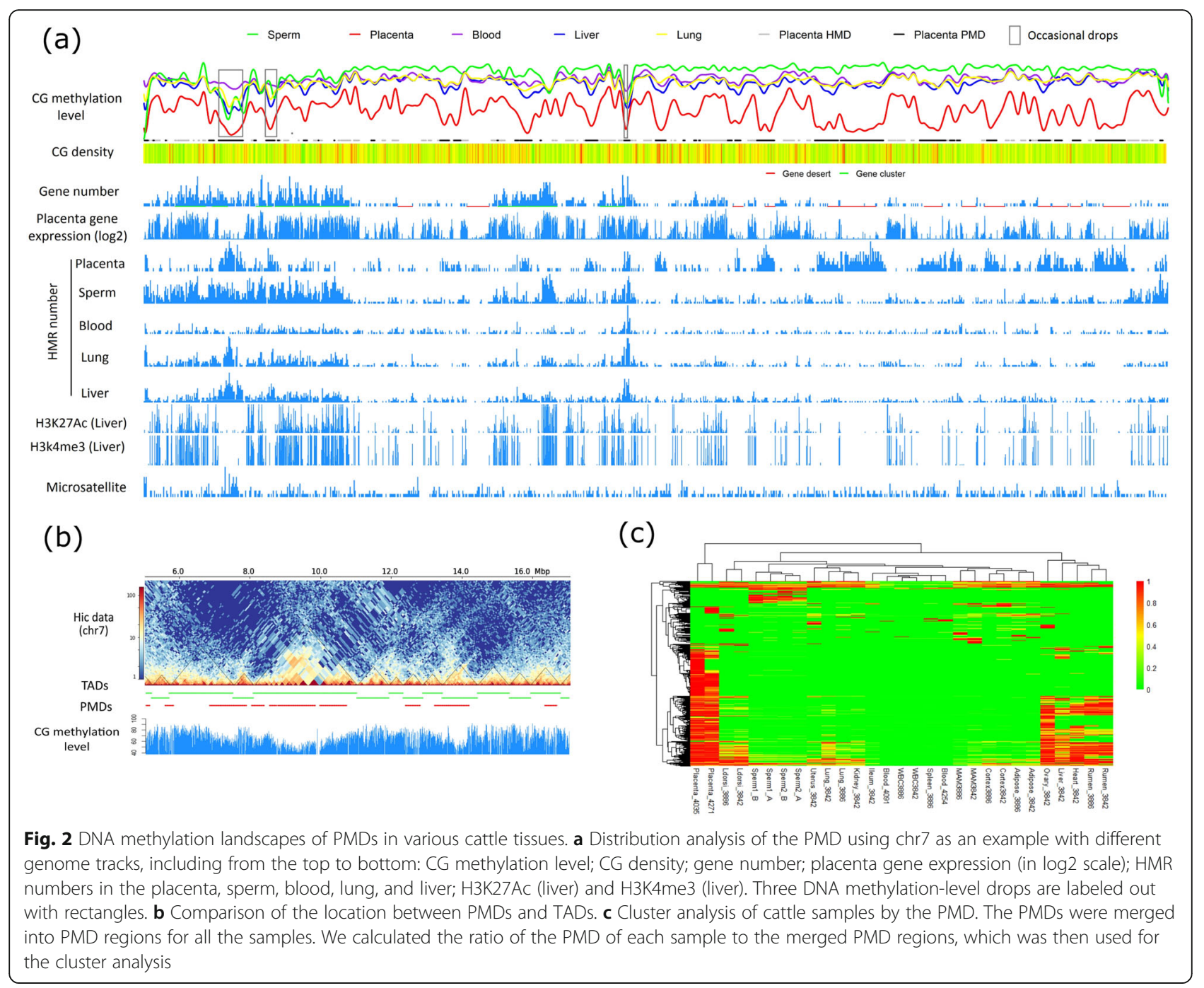

HMRs showed large differences in both location and size among the different clusters (Fig. 2a and Additional file 1: Figure S16). In somatic tissues and sperm, HMRs were highly enriched in the promoters, eCGIs, tRNAs, and satellites, while the placenta samples showed the opposite trend (Additional file 1: Figure S17). We divided the HMRs into two types according to their overlapping with TSS or not: TSS-HMR and non-TSS-HMR. The peak size of non-TSS-HMR for the placenta was around $5000 \mathrm{bp}$ and those for sperm and normal tissues were much smaller in size (around 500 bp) (Fig. 3a). However, interestingly, the peak sizes of TSS-HMR were highly consistent, centering around $2000 \mathrm{bp}$ among all samples (Fig. 3a). This might indicate the importance of the TSSHMR throughout tissue development.

We calculated and plotted the distances from the two HMR boundaries to the nearest TSS. Of note, we found that the center of the HMR was usually located in the downstream of the TSS (Fig. 3b). We examined the relationship between methylation and transcription, using a correlation analysis between the methylation levels of intragenic DMRs and the expression of the closest genes based on 20 methylome and transcriptome data derived from the same samples in cluster 3. As expected, high methylation in DMRs had a negative correlation with gene expression, and this negative correlation grew stronger around the transcription start site (Fig. 3c). The strong negative correlation was not only in the gene promoters, but also extended downstream of the promoter up to $8 \mathrm{~kb}$ away (Fig. 3c). This analysis shows that transcription is strongly associated with intragenic DMRs in the tissues we examined, in line with the similar observations in human methylomes [52]. Additionally, as expected, we found the TSS-HMR with high CG density showed a stronger negative correlation with gene expression than TSS-HMR with low CG density did (Fig. 3c). 


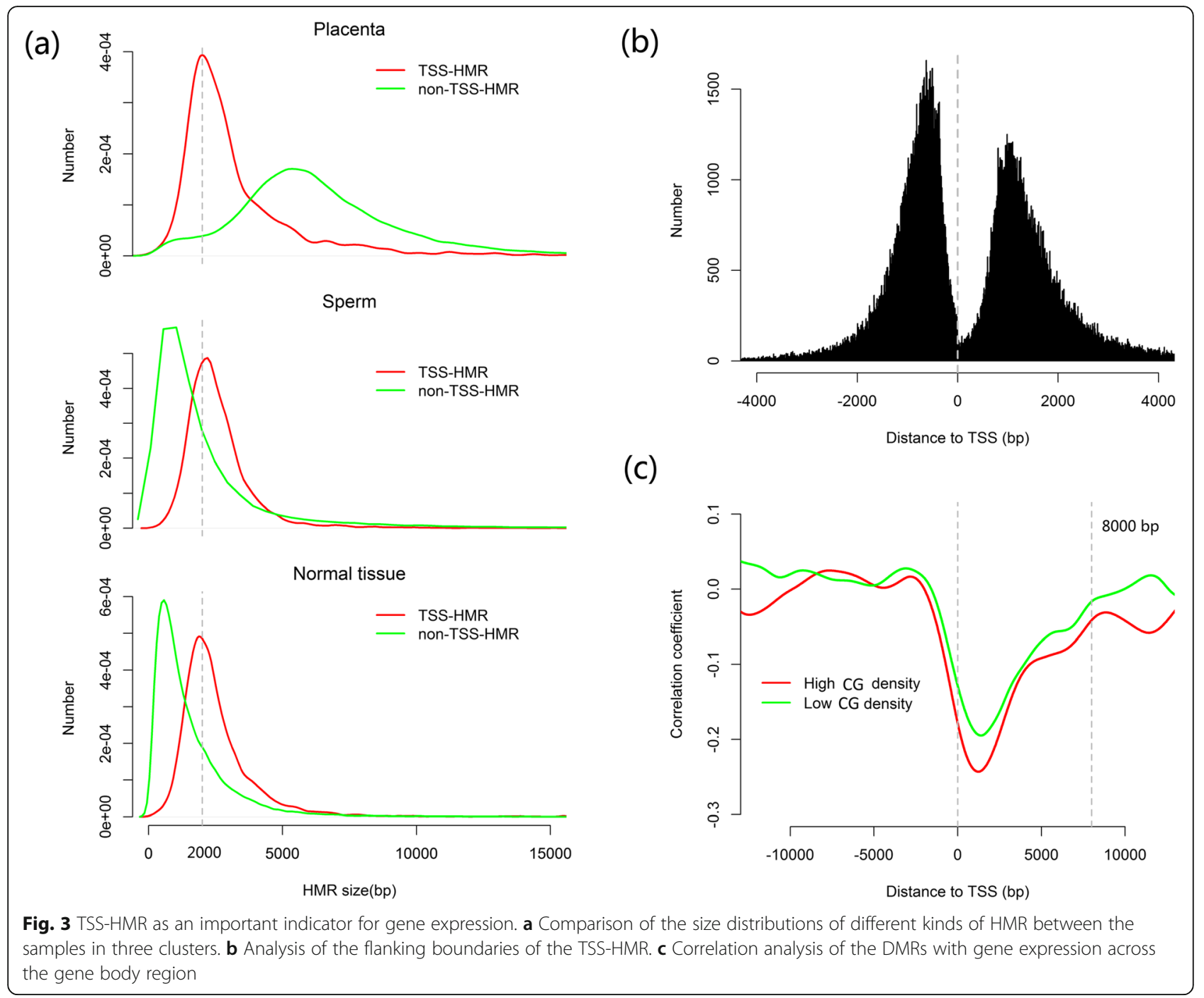

We then counted commonly and differentially methylated TSS-HMR for the homologous genes between cattle and human for either the liver or the kidney, as we did previously for sperm [47]. Over $90 \%$ genes with TSS-HMR were conserved (i.e., commonly methylated, either highly or lowly methylated) between cattle and human: $91.70 \%$ for the liver or $94.03 \%$ for the kidney. These liver and kidney genes with conserved methylated TSS-HMR were involved in basic biological processes, like RNA processing, protein folding, and cell cycle (Additional file 1: Figure S18). On the other hand, we did find 69 homologous genes with differentially methylated TSS-HMR (Additional file 2: Table S10). They included some lipid-related genes (e.g., CYP11A1, PLD6, MGLL, CYP39A1, RAB7A), which were hypomethylated in human. Human liver and kidney also shared 8 genes with hypomethylated TSS-HMR. However, their mechanisms are not well understood and require future investigations.
Classification of the TSS-HMR: tissue-specific TSS-HMR for cattle gene expression

To better understand the TSS-HMR, we classified genes into 5 groups according to their promoter location relative to the TSS-HMR by integrating the WGBS data of $27 \mathrm{di}-$ verse samples (except placentas as their methylation levels were lower) and their corresponding RNA-seq data. The 5 gene groups were as follows: (1) no-TSS-HMR: the gene with its TSS not located in the HMR in all samples; (2) total in HMR: the gene totally located in the HMR in at least one sample; (3) TSS-HMR T1: only one gene with its TSS located in the HMR in at least one sample; (4) TSSHMR T2: two opposite strand genes with their TSS located in the same HMR; and (5) TSS-HMR T3: two or multiple transcripts of one gene with TSSs located in different HMRs (Fig. 4a). According to this classification, we identified $20.46 \%$ of the annotated coding genes (NCBI) without TSS in all the samples. To avoid the possible incomplete gene annotation, we fine-mapped TSS for $1.84 \%$ 
(a)

(c)

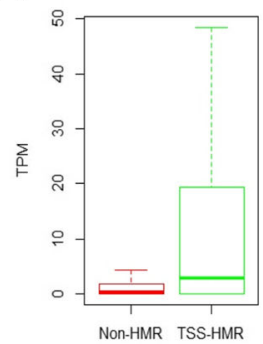

(g)

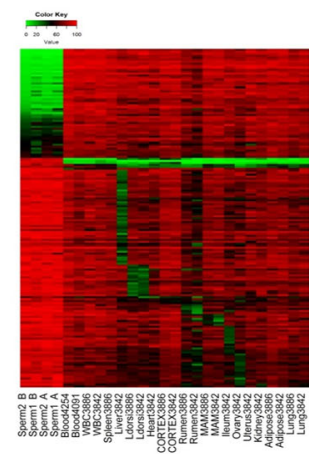

(d)

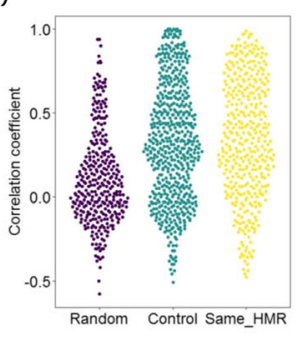

(h)

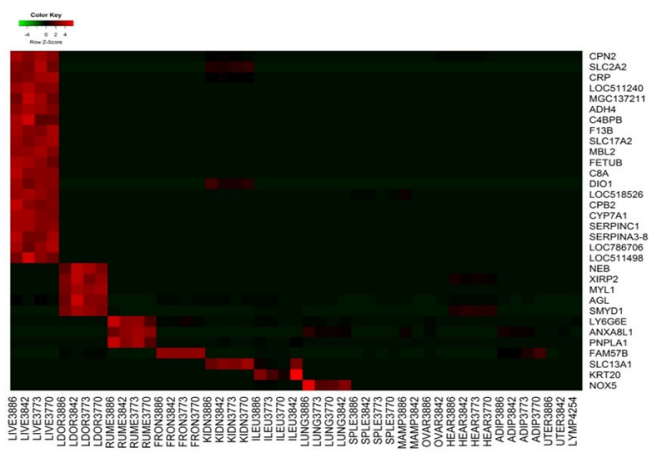

(e)

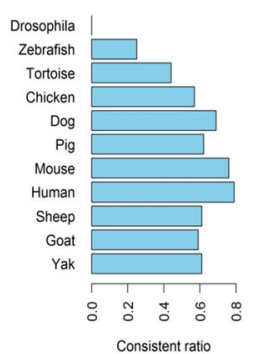

(b)

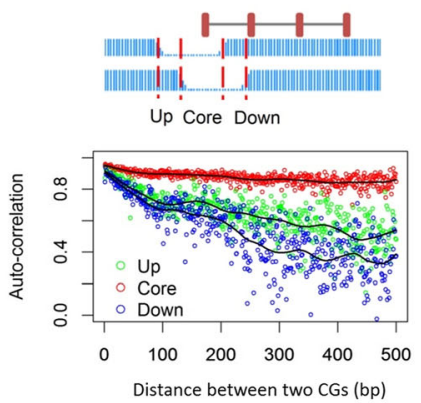

(f)

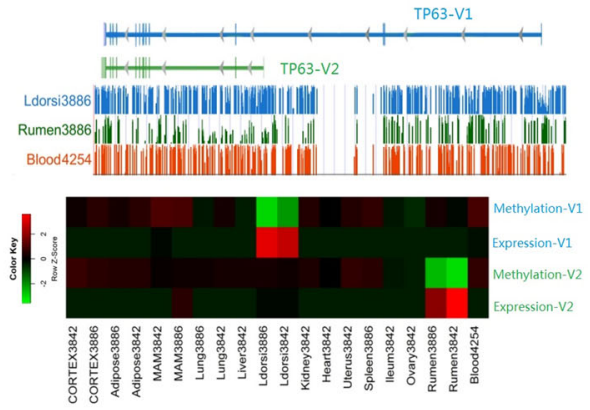

(i)

Fig. 4 Classifications of TSS-HMR and their tissue specificity. a Classification of the 5 types of the TSS-HMR: (1) no-TSS-HMR; (2) total in HMR; (3) TSS-HMR T1; (4) TSS-HMR T2; (5) TSS-HMR T3. Please refer to the main text for details. b Comparison of the methylation-level correlation of the CG with different distances between the TSS-HMR core region and two flank regions. c Boxplots of the gene expression for the genes with TSS-HMR or not. $\mathbf{d}$ Correlation analysis of the expression of the gene pairs with TSS located in the same HMR. Random: same number of genes pairs randomly chosen from all genes; control: same number of gene pairs randomly chosen from all genes with the same distance of the two TSS; Same_HMR: the gene pairs with TSS located in the same HMR. e The consistent ratio of gene pairs with TSS located in the same HMR of cattle in the other species, we defined the nearest two different strands genes as gene pairs. $\mathbf{f}$ The example of TP63: its tissue-specific expression of different transcripts from the two different TSS were regulated by the two tissue-specific TSS-HMRs. $\mathbf{g}$ Heatmap of the methylation level of tissuespecific TSS-HMR. $\mathbf{h}$ Heatmap of the expression levels of the genes showing tissue-specific high expression and tissue-specific TSS-HMR. $\mathbf{i}$ Transcription factor binding motif enrichment analyses of the tissue-specific TSS-HMR using the liver as an example

of genes using our RNA-seq data. Most (81.4\%) of annotated coding genes were classified as TSS-HMR genes, including TSS-HMR T1 (61.63\%), TSS-HMR T2 (7.59\%), and TSS-HMR T3 (4.57\%). The genes (7.60\%) totally within HMR were short in length (average length $=2551$ bp; medium length = 1726 bp) and enriched in G-proteincoupled receptor signaling pathway, sensory perception of smell, and nucleosome assembly (FDR $<0.01)$ (Additional file 2: Table S11).
As HMR boundaries varied among different tissues, we then investigated its core and flanking regions. Compared to the two upstream and downstream regions of the HMR, the core regions (shared by the TSS-HMR in all samples) had much higher CG density and were more conserved in terms of the methylation level among different tissues (Additional file 1: Figure S19). The correlation efficient values between the methylation levels of adjacent CGs were kept stably high (>0.8) even for long 
distance in the HMR core region while those of the HMR flank regions decreased more rapidly (Fig. 4b). Thus, to study the relationship between methylation and gene expression, we focused on the core region. As expected, the expression of the genes that classified as non-TSS-HMR were mostly suppressed (Fig. 4c).

By performing correlation analyses between the paired genes' expressions, we found the paired genes (twingenes) within TSS-HMR T2 would have greater chances of being co-expressed (Fig. 4d). Moreover, we examined the existence of those paired genes in other species. The paired genes were more consistently co-expressed among different mammals and chicken but showed lower consistent ratios in other species, including tortoise, zebrafish, and Drosophila (Fig. 4e). For example, we performed similar TSS and HMR analyses using human liver and kidney WGBS datasets. We found 783 such gene pairs in human and $514(65.65 \%=514 / 738$, Additional file 2: Table S12) of them with their TSS overlapped with human HMRs. Hence, this cross-species comparison between cattle and other species revealed the important roles of epigenome evolution in mammals and chicken.

As for genes within the TSS-HMR T3, they have a possibility to be regulated by tissue-specific methylation of TSS-HMR. For an example, the TP63 gene could be expressed from two different TSSs and their expressions were tissue-specifically regulated by the methylation levels of these two TSS in LD muscle and rumen, respectively (Fig. 4f). We recovered 122,867 cases, involving 4123 genes with different TSS-HMRs in at least two samples and 171 tissue-specific TSS-HMRs (Fig. 4g). We also identified 3207 genes contained by 12 modules that specifically highly expressed in 12 different tissues by performing a weighted gene co-expression network analysis (WGCNA) (Additional file 1: Figure S20). Those genes were enriched highly in GO terms related to the special tissue functions (Additional file 2: Table S13). Combining with the gene expression using the RNA-seq data, we found tissue-specific HMRs for 32 genes were highly correlated with their expression (Fig. 4h). For example, our results showed that liver-specific TSSHMR for the following genes: CPN2, SLC2A2, CRP, LOC511240, MGC137211, ADH4, C4BPB, F13B, SLC17A2, MBL2, FETUB, C8A, DIO1, LOC518526, CPB2, CYP7A1, SERPINC1, SERPINA3-8, LOC786706, and LOC511498. After filtering out 4 predicted LOC genes, we queried the left 16 genes against human GTEx portal [55] and cattle gene atlas [56]. Except for 3 genes, all other 13 genes were uniquely express in liver tissues of both human and cattle. MGC137211 and SERPINA3-8 were still uniquely expressed in the cattle liver, but they were not found in the human genome. On the contrary, DIO1 was not found in the cattle genome, and its expression in human thymus was higher than that in human liver. In summary, these cross-species comparisons revealed conserved tissue-specific gene expression were associated with conserved tissue-specific TSS-HMRs. This observation generally agreed with a recent report that conserved tissue-specific transcriptions across species could be more often explained by conserved tissuespecific DMRs [57]. Finally, we found that the tissuespecific TSS-HMRs were strongly enriched for putative binding sites of transcription factors which are known to have tissue-specific function (Fig. 4i). For example, we detected HNF6, PPARA, and Foxa2, which are liver-specific transcription factors and further confirm our above speculations.

\section{eCGI as a guard to avoid C/T mutation for the TSS-HMR core region}

Previous human studies have shown that the computational annotations of CGI (cCGI) suffer from inaccuracies [17]. Here, we totally identified 50,023 experimentally supported CGIs (eCGIs) at a single-base resolution using 27 whole genome bisulfite sequencing data (not including the two placentas) in cattle (Additional file 1: Figure S21, Additional file 1: Figure S22a, and Additional file 2: Table S14). The neCGI (cCGI not overlapped with eCGI) almost lost and even showed an opposite methylation patterns as compared to those of the eCGI (Additional file 1: Figure S22b). More importantly, we found that the genomic distributions of these eCGIs across chromosomes correlated more strongly with gene contents than with chromosome lengths (Additional file 1: Figure S23). This suggested that these hypomethylated regions might contain regulatory elements for gene expression. Furthermore, eCGI was highly enriched around the TSS as compared to the neCGI, which was actually highly enriched in the telomere of the chromosome (Fig. 5a, Additional file 1: Figure S24). We found eCGI overlapped with 10,503 (62.51\%) the TSS-HMR core region, which is consistent with the previous notion of the importance of CGI in the regulation of gene expression [58]. Earlier results also showed that the high methylation of the cytosine within CGI usually leads to $\mathrm{C} / \mathrm{T}$ mutations more easily [59]. We found that the eCGI was not only kept low in methylation but also its methylation level was more conserved among different tissues (samples) (Fig. 5b). We checked the distribution of the $\mathrm{C} / \mathrm{T}$ heterozygote (including $\mathrm{C} / \mathrm{T}, \mathrm{G} / \mathrm{A}$ ) for the eCGI in the two individuals using their genome sequencing data. The $\mathrm{C} / \mathrm{T}$ heterozygote rate was lower for all eCGI or the eCGI in the TSSHMR core region (Additional file 1: Figure S25). We searched for motifs enriched in the neCGI as compared to eCGI. As expected, we found more motifs of TG or 

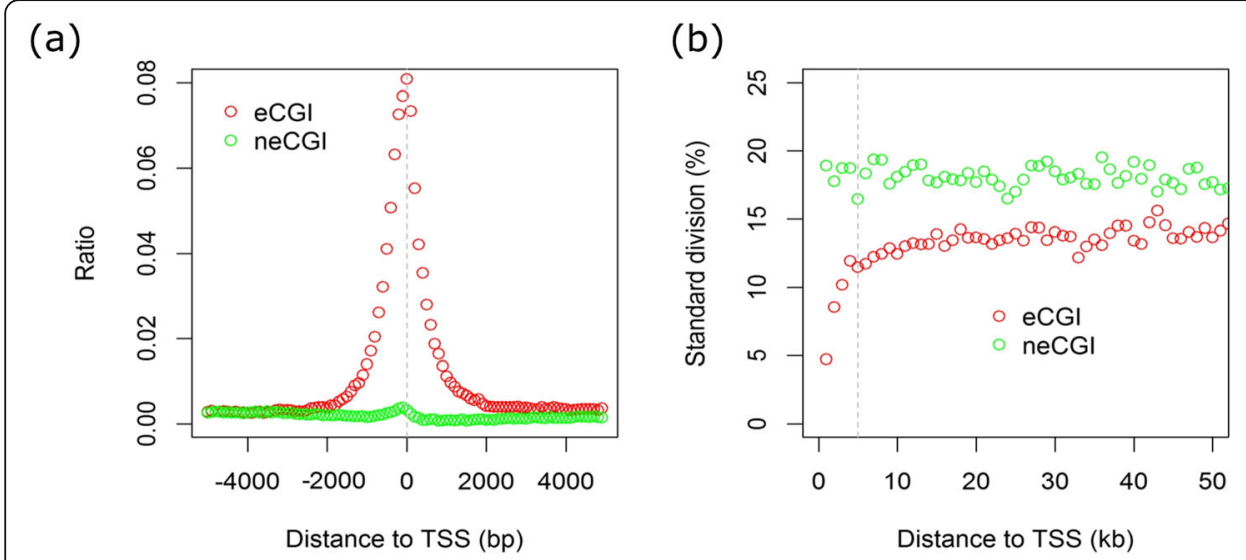

(c)

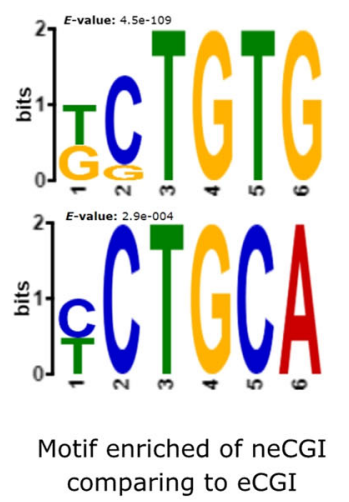

Fig. 5 Comparison between the eCGI and neCGI. a eCGI was enriched around the TSS. b The eCGI was lowly methylated and conserved among different tissues (samples), shown as the standard division of methylation levels among the different samples diminished as their distances to the TSS decreased. c Motif enrichment analyses of the neCGI as compared to the eCGl

GA, which were possibly the results of the mutation from CG to TG (Fig. 5c). This provided more reasons for the low methylation level in the eCGI, which might actually protect sequence from $\mathrm{C} / \mathrm{T}$ mutation in the TSS-HMR.

\section{Common repeats may regulate gene expression via differential DNA methylation of the newly introduced transcription factor binding sites (TFBS)}

Most of the common repeats, especially retrotransposons, showed high methylation levels, which repress their transcriptions (Additional file 1: Figure S27). But there are still some repeat elements hypomethylated ( $\leq$ $3.3 \%)$, which are highly enriched in the regions around the TSS (2000 bp) (Fig. 6a). We plotted their observed/ expected ratios for each repeat subclass (Fig. 6b). We checked whether common repeats would bring special sequences such as transcription factor binding sites (TFBS) to the TSS-HMR. Because repeat elements could be broken into short pieces because of multiple rounds of insertions, we focused on the full-length elements (integrity $>80 \%$ ) located in the TSS-HMR. In total, we found 4389 elements that dispersed in the TSS-HMR (Additional file 1: Figure S27). We searched for the specific sequences or motifs enriched in the different elements as compared to all TSS-HMR sequences. We observed that only Bov-A2 elements are enriched for multiple known Zinc-finger-related transcription factor binding motifs (Fig. 6c). As an example, we showed the results for a subset of young Bov-A2, which recently inserted into and split ancient common repeats (Fig. 6c). But we did not find any significant GO term for the genes containing Bov-A2 in the TSS-HMR. Interestingly, we detected several hypomethylated Bov-A2 around the TSS-HMR in sperm cells. For example, NME8, one known gene related to sperm function, containing one
Bov-A2 element insertion with 4 AZF1 binding motifs in its TSS-HMR, was especially hypomethylated in the sperm samples (Fig. 6d). We also found one young BovA2 element embedded in an old Bov-tA2 in the promoter region of the PBX4 (pre-B cell leukemia homeobox 4) gene, which encodes a member of the pre-B cell leukemia transcription factor family. Again, we detected low methylation of this TSS-HMR only in the sperm samples (Fig. 6d).

\section{Discussion}

Using WGBS, we generated one of the first largescale, single-nucleotide resolution cattle somatic tissue methylomes. Cattle-unique tissue-like rumen was also reported for the first time. The global CG methylation levels detected ranged from 72.8 to $78.1 \%$ among our cattle samples, which were similar to those in other mammalian species like humans ( 70\%) [21] and what we reported previously [24]. Our genome-wide cattle methylomes confirmed existing knowledge that DNA methylation is important for gene expression and plays a critical role in tissuespecific processes $[5,60]$. In promoter regions, DNA methylation is associated with transcriptional repression whereas in gene bodies, DNA methylation is generally enriched in the body of highly transcribed genes [61-64]. We tested the impacts of genome assembly quality on read mapping and DNA methylation calling, revealing DNA methylation peaks and valleys did change their locations and magnitudes, especially when they are near chromosome ends and sudden drops.

\section{PMDs}

In this study, we reported large-scale PMDs in multiple cattle tissues. We then cross-referenced them on the 


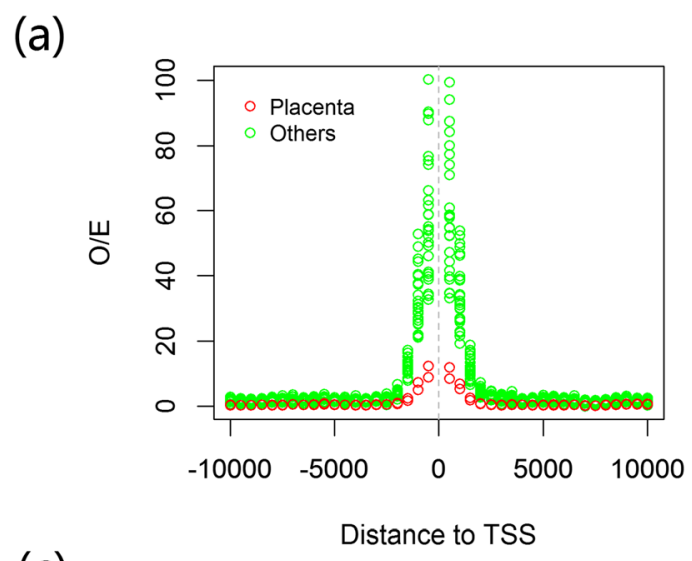

(c)

(b)

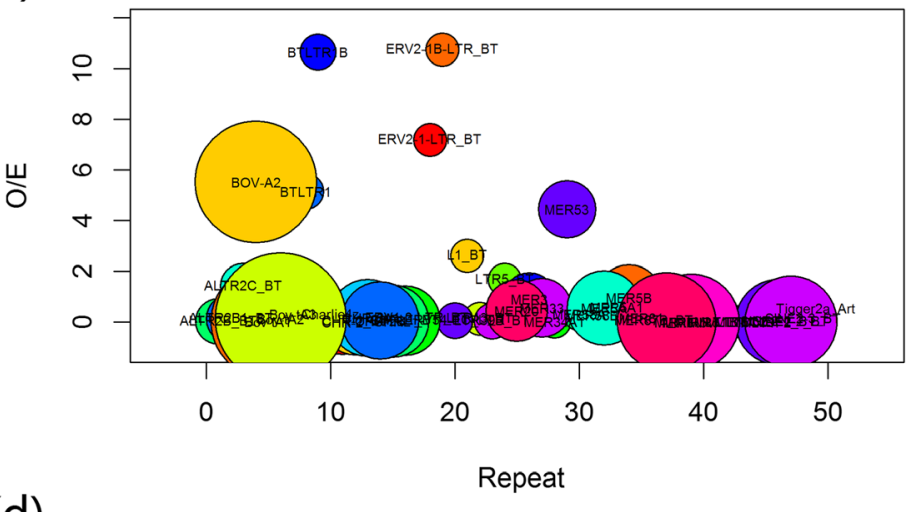

(d)
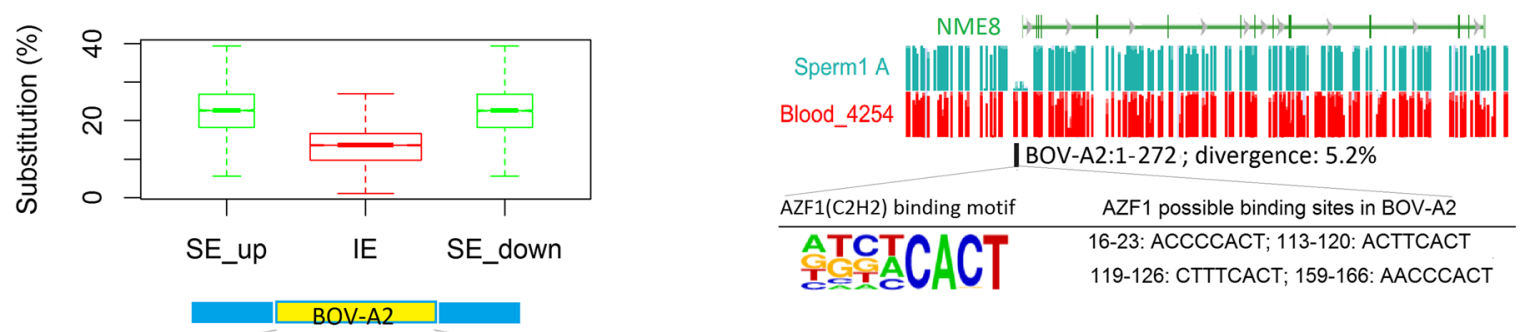

SeqBias: polyA-repeat; SeqBias: C/A-bias; SeqBias: polyC-repeat; SeqBias: G/A bias; SeqBias: GCW-triplet; Ik-1; SeqBias: CG bias; SeqBias: GA-repeat; Initiator;

AZF1; STZ; At5g04390; At3g60580; MYB88; Nkx2.2; Nkx2.1; Nkx2.5; Bcl6; Bapx1; PRDM10; AT1G69570; Knotted; PRDM1
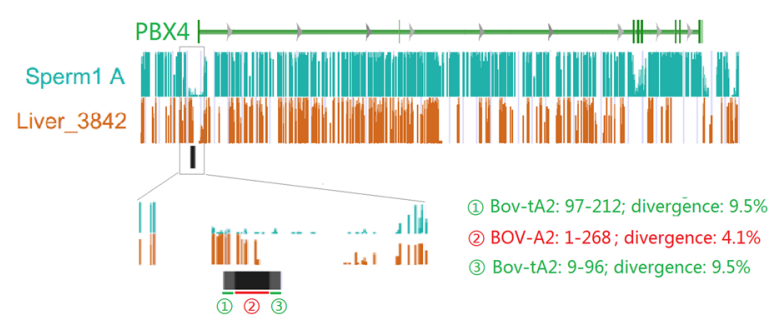

Fig. 6 Analysis of hypomethylated repeats. a Hypomethylated repeats were enriched around the TSS for different samples. b Analyses of the repeats located in the TSS-HMR; observed/expected ratio of the repeats (y-axis) plotted for each repeat subclass; the size: the number of the repeats located in the TSS-HMR. c Analysis of young Bov-A2 element insertions; SE_up: the upstream part of ancient repeats, SE_down: the downstream part of ancient repeats. IE, the young Bov-A2 elements, which inserted more recently, thus split ancient repeats. The $y$-axis represents the sequence divergences, thus age of repeats. BOV-A2Bov-A2. d NME8 and PBX4 as two examples for the Bov-A2 insertion in the tissues with different TSS-HMRs

chromosome level with $\mathrm{CpG}$, genes, transcriptions, HMRs, histone codes, satellites, and TADs. We found that cattle PMDs share features with those identified in other species, especially those identified in human tissues: localization in genomic regions with low GC contents, low CGI density, low gene density, and lack of active histone marks. Although PMDs have been associated with gene repression and inactive chromatin marks, genes within tissue-specific PMDs did display tissuespecific functions. Previous human results show that PMDs are established within preformed TAD B compartments after cell lineage decision in cardiac myocytes [65]. The higher order chromatin conformation is proposed to be a regulatory mechanism guiding cell typespecific establishment of CpG methylation and non-CpG methylation signatures, like PMDs in TAD B compartments and HMR in TAD A compartments, respectively.
Similarly, the endogenous bovine $\mathrm{Hi}-\mathrm{C}$ contact maps uncovered that TAD B compartments were often associated with PMDs in the cattle genome. Thus, we hypothesize that a similar silencing mechanism may operate in cattle PMDs during cattle tissue specification and development.

\section{HMRs}

We detected large differences between cattle somatic tissues in terms of HMRs. For example, the peak size of non-TSS-HMR for the placenta was significantly larger than those in sperm and normal tissues, while the peak sizes of TSS-HMR were highly consistent, ( $2000 \mathrm{bp})$ among all tissue (Fig. 3a). This might indicate the dramatic difference of the placenta as compared to the sperm and other somatic tissues and the importance of the TSS-HMR throughout all tissues. We also classified 
genes into 5 groups according to their promoter location relative to the TSS-HMR and studied their potential impacts on gene regulation and genome evolution. By performing correlation analyses between the paired genes' expressions, we found the paired genes (twin-genes) within TSS-HMR T2 would have more chances to be co-expressed (Fig. 4d). Moreover, our results showed that those paired genes were more consistent across mammalian species. As for genes within the TSS-HMR T3, i.e., with variable TSS or promoters, we found that they had a high possibility of being regulated by tissuespecific methylation of TSS-HMR. We used WGCNA to study gene networks based on pairwise correlations between their expressions and identified tissue-specific genes related to tissue functions. Combining with the gene expression using the RNA-seq data, we found 32 genes' tissue-specific HMRs were highly correlated with their expression. The tissue-specific TSS-HMRs were greatly enriched for putative binding sites of transcription factors, which are known to have tissue-specific function (Fig. 4i). Combined with gene expression using the RNA-seq data, we identified tissue-specific gene expression correlated with tissue-specific HMR. Additionally, using our WGBS data, we totally identified 50,023 eCGIs at a single-base resolution and validated $42.24 \%$ of the total cCGI.

\section{Common repeats}

In germ cells like sperm, common repeats are normally highly methylated. The conserved piRNA pathway has been proposed to be important for recognizing and silencing repeats in germ cells [66]. However, we still found more than expected HMRs that overlapped common repeats, suggesting some individual elements can evade piRNA-based silencing. Examining patterns of HMR-associated repeats is very informative. One possibility is that just like genes, young repeats contain promoters or regulatory regions and/or their TF binding and transcription activation can facilitate their evading default methylation. Although most of Bov-A2 elements follow the normal expectation, showing a negative correlation between methylation level and age (represented by their divergence from its consensus sequence), we detected that some Bov-A2 elements were hypomethylated in cattle sperm cells. Similar to the young Alu subfamilies, which introduce binding sites for transcription factor SABP in human sperm $[67,68]$, we found some BovA2 elements inserted into genes like NME8 and PHX4 that function in spermatogenesis or transcription regulation. Through examining these Bov-A2 insertions, we found the binding sites for multiple AZF1 (azoospermia factor 1), which have an essential meiotic function in fly and human spermatogenesis [69]. Diseases associated with AZF1 include azoospermia and varicocele [70]. As the introduction of TFBS by active Bov-A2 insertions could change the promoter structure, we hypothesize that Bov-A2 insertions in sperm cells may be involved in specific regulation of functional genes.

\section{Future directions and limitations}

Genome editing technologies, CRISPR/Cas9, can directly target and edit individual methylation sites and therefore determine the exact function of DNA methylation at a specific site, as reviewed recently [71]. It is noted that because our data were produced from bulk cells, we were unable to determine the impact of cell composition on our results. Based on 64 human reference cell types, the human GTEx Consortium recently used the xCell method [72] to characterize the effect of cell type heterogeneity on analyses from bulk tissue [55]. Estimated cell type abundances from bulk RNA-seq across tissues reveal the cellular specificity of genetic regulation of gene expression across human tissues [73]. Due to limited resources, such as cattle reference cell types, future studies will be warranted to test these hypotheses and estimate their effects.

\section{Conclusions}

In summary, using conventional WGBS and RNA-seq, we provided baseline methylation and transcription profiles for cattle somatic cells at a single-base resolution. We characterized the DNA methylome and assessed DNA methylation patterns. We reported rich data sets of PMDs and HMRs across different tissues and detected that some of them were correlated with tissue development. Our study contributes to the understanding of cattle DNA methylation patterns and provides foundational information for further investigations.

\section{Methods \\ Sample collection, DNA and total RNA isolation, and sequencing}

In this study, we collected 16 tissue types under the approval of the US Department of Agriculture, Agricultural Research Service, Beltsville Agricultural Research Center's Institutional Animal Care and Use Committee (Protocol 16-016). Tissues were collected, snap frozen in liquid $\mathrm{N}_{2}$ immediately after excision, and kept at $-80^{\circ} \mathrm{C}$ until use. Ten published samples were described before [24], including parenchymal tissue from the mammary glands, whole blood cells, and prefrontal cortex of the brain collected from two healthy adult Holstein cows (3-4 years old; one lactating and one non-lactating). Semen straws were collected twice from two fertile Holstein bulls. Among the newly generated data, we collected additional samples from the same two Holstein cows and their similar relatives based on a similar list as described before (Harhay et al., 2010). Genomic DNA 
for lung tissue was isolated according to the QIAamp DNA Mini Kit protocol (QIAGEN, Valencia, CA, USA). The quality of DNA samples was evaluated using the 2100 Bioanalyzer (Agilent Technologies, Santa Clara, CA, USA) including degradation, potential RNA contamination, purity (OD260/OD280), and concentration using a spectrophotometer (NanoDrop Technologies, Rockland, DE) to meet the requirements for library construction. We extracted the total RNA from snap-frozen tissues using TRIzol (Invitrogen, Carlsbad, CA, USA) according to the manufacturer's instructions. We measured the quantity and purity of RNA using a NanoDrop 8000 Spectrophotometer (NanoDrop Technologies, Wilmington, DE) and Agilent 2100 Bioanalyzer System (Agilent Technologies). We contracted Novogene USA (Sacramento, CA, USA) to sequence these RNA samples using the Illumina HiSeq 2000 platform (Illumina, San Diego, CA) with paired-end (100 to150 bp) reads (Additional file 2: Table S1).

\section{WGBS library construction, sequencing, and identification of methylcytosine}

The qualified genomic DNA from all samples were used to construct libraries. Briefly, $3 \mu \mathrm{g}$ of genomic DNA spiked with unmethylated lambda DNA was fragmented into 200-300 bp using a Covaris S220 (Covaris, Inc., Woburn, MA, USA), followed by terminal repairing and A ligation. Different cytosine methylated barcodes were ligated to sonicated DNA for different samples. The DNA bisulfite conversion was performed using the EZ DNA Methylation Gold Kit (Zymo Research, Irvine, CA, USA). Then, single-stranded DNA fragments were amplified using the KAPA HiFi HotStart Uracil + ReadyMix (2 X) (Kapa Biosystems, Wilmington, MA, USA). The library concentration was quantified using a Qubit 2.0 fluorometer (Life Technologies, Carlsbad, CA, USA) and qPCR (iCycler, BioRad Laboratories, Hercules, CA, USA), and the insert size was checked using the Agilent 2100. To decrease the batch effect, the libraries for one sample were balanced, mixed with other libraries with different barcodes, and sequenced on different lanes of a HiSeq X Ten platform to generate 150-bp paired-end reads by Novogene (Novogene, Beijing, China).

Programs FastQC v 0.11.2 (FastQC) and Trim Galore v 0.4.0 (Trim Galore) were used to generate sequence quality reports and to trim/filter the sequences, respectively. For each sample, high-quality reads were obtained after trimming low-quality bases and the adapter sequences. The cleaned data for each sample were merged and aligned to the reference genome (ARS-UCD1.2) using bowtie 2 under the Bismark software (0.14.5) with the parameters -p 3 -N 1 -D 20. The methylcytosine information was extracted using the bismark_methylation extractor after deduplicating the duplication reads. The first $6 \mathrm{bp}$ were ignored for the paired-end reads to decrease the potential effects of severe bias toward nonmethylation in the end-of-reads caused by end repairing.

\section{Genome sequencing library construction, sequencing, and identification of SNP}

The lung DNA samples of the two Holstein cows were sequenced using the Illumina NextSeq550 platform, with the Nextera library preparation and sequence generation according to the manufacturer's protocols. NGSQCToolkit v2.3.3 was used to trimmed adapter sequences and lowquality reads. All the clean reads were mapped on the reference genome (ARS-UCD1.2) using BWA v0.7.12 software. We only used the reliable mapped reads for SNP calling. The SNP positions within the aligned reads compared to the reference genome were detected using the pileup function in SAMtools v.1.7 utilities. SNPs were predicted with a minimum mapping quality $(-\mathrm{Q})$ of 20 and with the minimum and maximum read depths of 3 and 100, respectively.

\section{RNA sequencing read alignment and assembly}

The total RNA was first treated with DNase I to remove residual DNA. Then, poly(A) mRNA was isolated using beads with oligo(dT). The purified mRNA was first fragmented using the RNA fragmentation kit. First-strand cDNA synthesis was performed using random hexamer primers and reverse transcriptase. After the first strand was synthesized, a custom second-strand primer and strand synthesis buffer (Illumina) were both added, followed by dNTPs, RNase $\mathrm{H}$, and DNA polymerase I to start the second-strand synthesis. Second, after a series of terminal repair, A ligation, and sequencing adaptor ligation, the double-stranded cDNA library is completed through size selection and PCR enrichment. Then, the cDNA libraries were prepared according to Illumina's protocols and sequenced on the Illumina platform in Novogene USA, Sacramento, CA.

NGSQCToolkit v2.3.3 was used to trimmed adapter sequences and low-quality reads. The clean reads were aligned on the reference genome (ARS-UCD1.2) [51] along with annotated genes in the NCBI (ftp://ftp.ncbi. nlm.nih.gov/genomes/all/GCF/002/263/795/GCF_002263 795.1_ARS-UCD1.2) using the HISAT2 v2.1.0 with the default parameters. The spliced reads were initially assembled to transcripts using the StringTie v1.3.3 software for each sample. Transcripts from all samples were merged to create a consensus reference transcriptome. The transcript per million mapped reads (TPM) and raw counts that mapped to the corresponding transcripts were estimated using the StringTie program.

\section{CpG island identification and validation}

We used a distance-based algorithm to identify singlebase resolution for $\mathrm{CpG}$ island on the cattle genome, 
following the CpGcluster software as described [17]. We calculated the methylation level of each computerpredicted $\mathrm{CpG}$ island (cCGI) for each sample. Only the CpG island with at least 5 CG detected with more than $5 \times$ coverage for each sample was used for further experimental validation. The eCGI was defined as methylation level less than $30 \%$ in at least one sample.

\section{Histone mortification localization analysis for the cattle liver}

ChIP-seq data for the cattle liver was downloaded from the Gene Expression Omnibus database with the accession number PRJEB6906. The sample preparation procedure can be found in [74]. NGSQCToolkit (version 2.3.3) software was used to filter the adapters and lowquality reads. Then, the qualified reads were aligned to the reference genome (ARS-UCD1.2) using bowtie2 (version 2.3.3; -N 0 -L 22 -i S,1,1.15 -dpad 15 -gbar 4), and peaks were called using MACS (version 1.4.2; -keep-dup 1 -wig -single-profile - space $=10$-diag) with default parameters.

\section{Identification of TAD using $\mathrm{Hi}-\mathrm{C}$ data}

The Hi-C data was retrieved from NCBI Sequence Read Archive under the accessions: SRR5753600, SRR5753603, and SRR5753606. These Hi-C libraries were prepared from the sequenced Hereford cow (Dominette) lung tissue and sequenced on an Illumina HiSeq 4000. NGSQCToolkit v2.3.3 was used to trimmed adapter sequences and lowquality reads. The clean reads were mapped on the reference genome (ARS-UCD1.2) using BWA software with parameters of mem -A1 -B4 -E50 - L0 -t 16. Hi-C matrices were imported to HiCExplorer v3.4.1 with the applications hicFindTADs and hicPlotTADs. Interaction frequency matrices at 50-kb resolution were transformed into $z$-score matrices based on the distribution of contacts at given genomic distances. The false discovery rate (FDR) was used to correct $P$ values with threshold of 0.01 . hicPlotTADs was used to plot specific regions for the interaction frequency matrices in combination with TAD boundary start and stop positions.

\section{PMD and HMR identification}

We utilized the methpipe software (http://smithlabresearch.org/downloads/methpipe-manual.pdf) to identify PMD by applying an HMM model to each sample. To detect the large PMDs accurately, we used different window sizes $(5 \mathrm{~kb}, 10 \mathrm{~kb}, 20 \mathrm{~kb}, 50 \mathrm{~kb})$ to generate the PMD localizations and examined the result by randomly selected visualization as recommended by the manual of the methpipe software. Finally, we chose 10-kb window size for all the samples. The HMR was identified following the manual of the methpipe software with the default parameters.

\section{Global comparison between methylomes of different} samples

The common CGs with depth greater than $10 \times$ among all samples were used for global comparison between each of the two sample pairs. Detections of DMC and DMR were applied using an $\mathrm{R}$ package (methykit, $\mathrm{R}$ version 3.3.3). The DMCs were defined as the methylation difference greater than $30 \%$ and $q$ value $<0.05$. The DMRs were defined as the average methylation difference greater than $30 \%$ and $q$ value $<0.05$ using a 500-bp window size. To receive more accurate DMRs, we only selected the DMRs that supported by at least 5 DMCs in the same direction for analyses (Additional file 2: Table S3 Column D). To call the tissue-specific DMRs, we ranked all DMRs by their frequencies derived from all pairwise comparisons. We then chose the top $0.01 \sim 0.3 \%$ of the DMRs (by considering the GO enrichment results) and merge them into the final nonredundant tissue-specific DMRs, which showed significant differences between the tissue to all other samples.

The genome structure annotation files for genes and repeats were downloaded from the NCBI database (ftp:// ftp.ncbi.nlm.nih.gov/genomes/all/GCF/002/263/795/ GCF_002263795.1_ARS-UCD1.2) [51]. The promoter regions were defined as $\pm 1000 \mathrm{bp}$ around the transcript start sites. The methylation levels for each element in different genomic features were calculated as the average methylation level of the CGs with at least $5 \times$ coverage. Only the elements that met the following criteria were used for further analysis: at least 10\% CG detection rate for elements with more than 50 CGs and at least five CGs detected for elements with fewer than 50 CGs. $R$ packages were used to plot the comparison results.

\section{Gene function analysis}

Gene functional annotation analyses were applied using the online DAVID software. The Fisher exact test was used to measure gene enrichment in annotation terms. $P$ values were corrected by FDR to search for significantly enriched terms. We used Homer software to detect enriched motifs within the tissue-specific TSS-HMR of genes. The MEME online software (http://meme-suite. org/) [75] was used to enrich the significant different motif between the neCGI and eCGI.

\section{Supplementary information}

Supplementary information accompanies this paper at https://doi.org/10. 1186/s12915-020-00793-5.

\footnotetext{
Additional file 1: Figure S1. Comparison of methylation distribution between two different cattle genome reference assemblies using a sperm sample as an example. Blue line: UMD3.1.1; Green line: ARSUCD1.2, from top left to down right: chr1-chr29. Figure S2. Comparison of methylation distribution between two different cattle genome reference assemblies using chr28 as an example for all samples. Blue line:
} 
UMD3.1.1; Green line: ARS-UCD1.2. Figure S3. Principal component analysis (PCA) for all samples using DNA methylation levels of $500 \mathrm{bp}$ windows: a, PC1 vs. PC2; b, PC1 vs. PC3; and c, PC1 vs. PC4. Figure S4. Correlation analyses for all samples using DNA methylation. Figure $\mathbf{S 5 .}$ Correlation analyses of DNA methylation using different window size. 20 $\mathrm{K}, 100 \mathrm{~K}, 500 \mathrm{~K}$ and $1 \mathrm{M}$ refer to window sizes of $20 \mathrm{~kb}, 100 \mathrm{~kb}, 500 \mathrm{~kb}$ and $1 \mathrm{Mb}$, respectively. Figure $\mathbf{S 6}$. Standard division of methylation level across all samples. We defined methylation conserved and variable regions as the bottom and top tails, respectively. Figure S7. Genome features enrichment in hypermethylation conserved regions, including genic region and neCpG island. Figure S8. Gene ontology analyses for the genes located in the methylation level conserved and variable regions. (a) Genes located in the hypermethylation conserved regions; (b) Genes located in the sperm hypomethylation variable regions; (c) Genes located in the sperm hypermethylation variable regions. Figure S9. Genome features enrichment in hypomethylation conserved regions, including promoters, eCpG islands, and tRNA genes. Figure S10. Genome features enrichment in methylation variable regions, including promoter and eCpG island. Note: the enrichment of the $C C p G$ island is mainly caused by eCpG island. Figure S11. GO analysis for the genes overlapped with methylation variable regions. Figure $\mathbf{S 1 2}$. Heatmap analysis using the methylation variable regions. Figure S13. Comparison of gene expressions between genes located in the PMD and non-PMD. Figure S14. Placenta HMDs, as compared to placenta PMDs, were enriched for the gene-related features including genic regions, promoters, eCGl and RefGenes. Figure S15. Comparison of methylation patterns for the gene and the CGl regions between HMD and PMD. Placenta HMDs showed significantly lower methylation patterns around the gene TSS and the CGI, while placenta PMDs were almost indistinguishable from the flanking backgrounds because of their low methylations. Figure S16. Comparison of HMR in terms of location and size among the different clusters. Figure S17. Genome feature enrichment in the HMRs of different clusters. Figure S18. Liver (a) and kidney (b) genes with conserved methylated TSSHMR between cattle and human were involved in basic biological processes, like RNA processing, protein folding and cell cycle. Figure S19. Comparison of the TSS-HMR core region and the TSS-HMR two flank regions in terms of (a) the CG density; (b) standard deviations of the methylation level; (c) DMC distribution; and (d) methylation level. Figure S20. WGCNA analysis for the RNA sequencing data. (a) cluster dendrogram of the gene expression; (b) heatmap plot for the expression of tissue specific high expression genes. Figure S21. Genome distribution of the eCpG island. (a) Distribution of the eCpG island on the 29 cattle chromosomes. (b) Genomic features enrichment in eCpG island, including promoter (1000 bp around the TSS) and the first exon. Figure S22. The methylation pattern between the eCGI and the neCGl. (a) heatmap of the cCGI methylation level for all samples; the blue bar: eCGl; the green bar: neCGl; (b) comparison of the methylation patterns between the eCGl and the neCGI. Figure S23. Correlation analysis for eCGls with (a) gene contents and (b) chromosome lengths. Figure S24. Comparison of the distribution on chromosomes between eCGI and the neCGl; red line: eCGl; green line: neCGl. Figure S25. CT heterozygote rate for the two animals in different CGIs; CT heterozygote represent CT and GA heterozygote when consider the two strands of DNA. Figure S26. Methylation level distributions for different genome features and samples. Figure S27. Distributions of 4389 common repeats located in the TSS-HMR.

Additional file 2: Table S1A. Sample information for the WGBS; Table S1B. Sample information for the RNA sequencing; Table S1C. Sample information for the whole genome sequencing; Table S2. Comparison of methylation statistics between two different cattle reference assemblies. Table S3. DMC and DMR number for each comparison pairs. Please note that the numbers, which may be affected by different common data amount for each tissue. Table S4. PMD information for different samples. Table S5. Placenta PMD percentages and genes located in the shared and lineage-specific placenta PMDs between cattle and human. Table S6. GO analyses for genes shared or specific in PMDs between human placenta and cattle placenta. Table S7. Gene ontology analysis for genes overlapping with cattle placenta HMD. Table S8. GO analysis for the genes overlapped with cattle placenta PMDs. Table $\mathbf{S 9 .}$ Information of 16 non-blood DNA methylation level drops. Table S10. Differentially methylated TSS-HMR between human and cattle. Table
S11. Gene ontology analysis for genes totally located in the HMR. Table S12. common twin genes in one HMR core region between cattle and human. Table S13. Gene ontology analyses for genes specifically high expressed in different tissues. Table S14. Information for experimentally supported CpG Islands.

\section{Abbreviations}

CGI: CpG island; DMC: Differentially methylated cytosine; FAANG: Functional Annotation of Animal Genome project; FDR: False discovery rate; GO: Gene Ontology; HMR: Hypomethylated region; LINE: Long interspersed nuclear element; LTR: Long terminal repeat; PMD: Partially methylated domain; O/ E: Observed/expected; RRBS: Reduced representation bisulfite sequencing; SINE: Short interspersed nuclear element; TFBS: Transcription factor binding site; TSS: Transcription start site; WGBS: Whole genome bisulfite sequencing

\section{Acknowledgements}

We thank Reuben Anderson, Mary Bowman, Donald Carbaugh, Christina Clover, Sarah McQueeney, Mary Niland, Alexandre Dimtchev, and Research Animal Services for the technical assistance. Mention of trade names or commercial products in this article is solely for the purpose of providing specific information and does not imply recommendation or endorsement by the US Department of Agriculture (USDA). The USDA is an equal opportunity provider and employer.

\section{Authors' contributions}

$Y Z$ and GEL conceived and designed the experiments. EEC, CL, RLB, JBC, and LY collected samples and/or generated NGS data. YZ, SL, YH, LF, YG, HX, SGS, $\mathrm{BDR}, \mathrm{CPVT}$, and LM performed in silico prediction and computational analyses. YZ and GEL wrote the paper. All authors read and approved the final manuscript.

\section{Funding}

This work was supported in part by the Fundamental Research Funds for the Central Universities (2662017QD016); National Natural Science Foundation of China (31902148); Natural Science Foundation of Hubei Province of China (2018CFB363); USDA National Institute of Food and Agriculture (NIFA) Agriculture and Food Research Initiative (AFRI) grant numbers 2013-6701520951, 2016-67015-24886, and 2019-67015-29321; and the US-Israel Binational Agricultural Research and Development (BARD) Fund grant number US-4997-17 from. This work was also supported in part by UDSA ARS appropriated project 8042-31000-001-00-D, 8042-31000-002-00-D, and 8042-31310078-00-D.

\section{Availability of data and materials}

All cattle RNA-seq (GSE137943) [76], WGBS (GSE147087) [77], and WGS data (GSE146345) [78] generated by this study are available from NCBI Gene Expression Omnibus (GEO; http://www.ncbi.nlm.nih.gov/geo/). Human WGBS datasets of the liver (SRR3269859), kidney, (SRR1654399, SRR1654400, and SRR1764401) [74], and placenta (SRX381710) [13] were downloaded from NCBI Sequence Read Archive, respectively.

\section{Ethics approval and consent to participate}

All samples were collected with approval of the US Department of Agriculture (USDA) Agriculture Research Service (ARS) Institutional Animal Care and Use Committee under Protocol 16-016. Consent to participate: not applicable.

\section{Consent for publication}

Not applicable

\section{Competing interests}

All authors declare no potential conflict of interest.

\section{Author details}

'Key Laboratory of Agricultural Animal Genetics, Breeding and Reproduction of Ministry of Education \& College of Animal Science and Technology, Huazhong Agricultural University, Wuhan 430070, China. ${ }^{2}$ Animal Genomics and Improvement Laboratory, Agricultural Research Service, USDA, Beltsville, MD 20705, USA. ${ }^{3}$ National Engineering Laboratory for Animal Breeding, Key Laboratory of Animal Genetics, Breeding and Reproduction of Ministry of 
Agriculture and Rural Affairs, College of Animal Science and Technology, China Agricultural University, Beiijing 100193, China. ${ }^{4}$ MRC Human Genetics Unit, Institute of Genetics and Molecular Medicine, University of Edinburgh, Edinburgh EH4 2XU, UK. ${ }^{5}$ Department of Animal and Food Sciences, University of Delaware, Newark, DE 19716, USA. ${ }^{6}$ Department of Animal and Avian Sciences, University of Maryland, College Park, MD 20742, USA.

\section{Received: 4 March 2020 Accepted: 12 May 2020}

\section{Published online: 06 July 2020}

\section{References}

1. Reik W, Dean W, Walter J. Epigenetic reprogramming in mammalian development. Science. 2001;293(5532):1089-93.

2. Morgan HD, Santos F, Green K, Dean W, Reik W. Epigenetic reprogramming in mammals. Hum Mol Genet. 2005;14(suppl 1):R47-58.

3. Sasaki $H$, Matsui $Y$. Epigenetic events in mammalian germ-cell development: reprogramming and beyond. Nat Rev Genet. 2008;9(2):129-40.

4. Igarashi J, Muroi S, Kawashima H, Wang X, Shinojima Y, Kitamura E, Oinuma T, Nemoto N, Song F, Ghosh S. Quantitative analysis of human tissuespecific differences in methylation. Biochem Biophys Res Commun. 2008; 376(4):658-64

5. Law JA, Jacobsen SE. Establishing, maintaining and modifying DNA methylation patterns in plants and animals. Nat Rev Genet. 2010;11(3):204-20.

6. Lokk K, Modhukur V, Rajashekar B, Märtens K, Mägi R, Kolde R, Koltšina M, Nilsson TK, Vilo J, Salumets A, et al. DNA methylome profiling of human tissues identifies global and tissue-specific methylation patterns. Genome Biol. 2014;15(4):3248

7. Feng S, Cokus SJ, Zhang X, Chen PY, Bostick M, Goll MG, Hetzel J, Jain J, Strauss SH, Halpern ME, et al. Conservation and divergence of methylation patterning in plants and animals. Proc Natl Acad Sci U S A. 2010;107(19): 8689-94.

8. Zemach A, McDaniel IE, Silva P, Zilberman D. Genome-wide evolutionary analysis of eukaryotic DNA methylation. Science. 2010;328(5980):916.

9. Varley KE, Gertz J, Bowling KM, Parker SL, Reddy TE, Pauli-Behn F, Cross MK, Williams BA, Stamatoyannopoulos JA, Crawford GE, et al. Dynamic DNA methylation across diverse human cell lines and tissues. Genome Res. 2013; 23(3):555-67.

10. Lister R, Pelizzola M, Dowen RH, Hawkins RD, Hon G, Tonti-Filippini J, Nery JR, Lee L, Ye Z, Ngo Q-M. Human DNA methylomes at base resolution show widespread epigenomic differences. Nature. 2009;462(7271):315-22.

11. Berman BP, Weisenberger DJ, Aman JF, Hinoue T, Ramjan Z, Liu Y, Noushmehr H, Lange CP, van Dijk CM, Tollenaar RA, et al. Regions of focal DNA hypermethylation and long-range hypomethylation in colorectal cancer coincide with nuclear lamina-associated domains. Nat Genet. 2011; 44(1):40-6.

12. Hon GC, Hawkins RD, Caballero OL, Lo C, Lister R, Pelizzola M, Valsesia A, Ye Z, Kuan S, Edsall LE, et al. Global DNA hypomethylation coupled to repressive chromatin domain formation and gene silencing in breast cancer. Genome Res. 2012;22(2):246-58.

13. Schroeder DI, Blair JD, Lott P, Yu HO, Hong D, Crary F, Ashwood P, Walker C, Korf I, Robinson WP, et al. The human placenta methylome. Proc Natl Acad Sci U S A. 2013;110(15):6037-42.

14. Kubo N, Toh H, Shirane K, Shirakawa T, Kobayashi H, Sato T, Sone H, Sato Y, Tomizawa S-l, Tsurusaki Y, et al. DNA methylation and gene expression dynamics during spermatogonial stem cell differentiation in the early postnatal mouse testis. BMC Genomics. 2015;16(1):624.

15. Salhab A, Nordström K, Gasparoni G, Kattler K, Ebert P, Ramirez F, Arrigoni L, Müller F, Polansky JK, Cadenas C, et al. A comprehensive analysis of 195 DNA methylomes reveals shared and cell-specific features of partially methylated domains. Genome Biol. 2018;19(1):150.

16. Lee CM, Barber GP, Casper J, Clawson H, Diekhans M, Gonzalez JN, Hinrichs AS, Lee BT, Nassar LR, Powell CC, et al. UCSC Genome Browser enters 20th year. Nucleic Acids Res. 2019;48(D1):D756-61.

17. Mendizabal I, Yi SV. Whole-genome bisulfite sequencing maps from multiple human tissues reveal novel $\mathrm{CpG}$ islands associated with tissuespecific regulation. Hum Mol Genet. 2015;25(1):69-82.

18. Gaszner M, Felsenfeld G. Insulators: exploiting transcriptional and epigenetic mechanisms. Nat Rev Genet. 2006;7(9):703-13.

19. Dhayalan A, Rajavelu A, Rathert P, Tamas R, Jurkowska RZ, Ragozin S, Jeltsch A. The Dnmt3a PWWP domain reads histone 3 lysine 36 trimethylation and guides DNA methylation. J Biol Chem. 2010;285(34):26114-20.
20. Zhang Y, Jurkowska R, Soeroes S, Rajavelu A, Dhayalan A, Bock I, Rathert P Brandt O, Reinhardt R, Fischle W, et al. Chromatin methylation activity of Dnmt3a and Dnmt3a/3L is guided by interaction of the ADD domain with the histone $\mathrm{H} 3$ tail. Nucleic Acids Res. 2010;38(13):4246-53.

21. Molaro A, Hodges E, Fang F, Song Q, McCombie WR, Hannon GJ, Smith AD. Sperm methylation profiles reveal features of epigenetic inheritance and evolution in primates. Cell. 2011;146(6):1029-41.

22. Qu J, Hodges E, Molaro A, Gagneux P, Dean MD, Hannon GJ, Smith AD. Evolutionary expansion of DNA hypomethylation in the mammalian germline genome. Genome Res. 2018;28(2):145-58.

23. Hammoud SS, Nix DA, Zhang H, Purwar J, Carrell DT, Cairns BR. Distinctive chromatin in human sperm packages genes for embryo development. Nature. 2009:460(7254):473-8.

24. Zhou Y, Connor EE, Bickhart DM, Li C, Baldwin RL, Schroeder SG, Rosen BD, Yang L, Van Tassell CP, Liu GE. Comparative whole genome DNA methylation profiling of cattle sperm and somatic tissues reveals striking hypomethylated patterns in sperm. GigaScience. 2018;7(5):giy039.

25. Adelson DL, Raison JM, Edgar RC. Characterization and distribution of retrotransposons and simple sequence repeats in the bovine genome. Proc Natl Acad Sci. 2009;106(31):12855-60.

26. Iwasaki YW, Siomi MC, Siomi H. PIWI-interacting RNA: its biogenesis and functions. Annu Rev Biochem. 2015;84:405-33.

27. Czech B, Hannon GJ. One loop to rule them all: the ping-pong cycle and piRNA-guided silencing. Trends Biochem Sci. 2016;41(4):324-37.

28. Bakshi A, Herke SW, Batzer MA, Kim J. DNA methylation variation of humanspecific Alu repeats. Epigenetics. 2016;11(2):163-73.

29. Bourc'his D, Bestor TH. Meiotic catastrophe and retrotransposon reactivation in male germ cells lacking Dnmt3L. Nature. 2004;431(7004):96-9.

30. Taiwo O, Wilson GA, Morris T, Seisenberger S, Reik W, Pearce D, Beck S, Butcher LM. Methylome analysis using MeDIP-seq with low DNA concentrations. Nat Protoc. 2012;7(4):617-36.

31. de Montera B, Fournier E, Shojaei Saadi HA, Gagne D, Laflamme I, Blondin P, Sirard MA, Robert C. Combined methylation mapping of $5 \mathrm{mC}$ and $5 \mathrm{hmC}$ during early embryonic stages in bovine. BMC Genomics. 2013;14:406.

32. Couldrey C, Brauning R, Bracegirdle J, Maclean P, Henderson HV, JC ME. Genome-wide DNA methylation patterns and transcription analysis in sheep muscle; 2014.

33. Gao F, Zhang J, Jiang P, Gong D, Wang J-W, Xia Y, Østergaard MV, Wang J, Sangild PT. Marked methylation changes in intestinal genes during the perinatal period of preterm neonates. BMC Genomics. 2014;15(1):716.

34. Huang Y-Z, Sun J-J, Zhang L-Z, Li C-J, Womack JE, Li Z-J, Lan X-Y, Lei C-Z, Zhang C-L, Zhao X. Genome-wide DNA methylation profiles and their relationships with mRNA and the microRNA transcriptome in bovine muscle tissue (Bos taurine). Sci Rep. 2014:4:6546.

35. Lee J-R, Hong CP, Moon J-W, Jung Y-D, Kim D-S, Kim T-H, Gim J-A, Bae J-H, Choi $Y$, Eo J. Genome-wide analysis of DNA methylation patterns in horse. BMC Genomics. 2014;15(1):598.

36. Su J, Wang Y, Xing X, Liu J, Zhang Y. Genome-wide analysis of DNA methylation in bovine placentas. BMC Genomics. 2014;15(1):12.

37. Shojaei Saadi HA, O'Doherty AM, Gagne D, Fournier E, Grant JR, Sirard MA, Robert C. An integrated platform for bovine DNA methylome analysis suitable for small samples. BMC Genomics. 2014;15:451.

38. Cao J, Wei C, Liu D, Wang H, Wu M, Xie Z, Capellini TD, Zhang L, Zhao F, Li L. DNA methylation landscape of body size variation in sheep. Sci Rep. 2015;5:13950.

39. Choi M, Lee J, Le MT, Nguyen DT, Park S, Soundrarajan N, Schachtschneider KM, Kim J, Park J-K, Kim J-H. Genome-wide analysis of DNA methylation in pigs using reduced representation bisulfite sequencing. DNA Res. 2015;22(5):343-55.

40. Schachtschneider KM, Madsen O, Park C, Rund LA, Groenen MA, Schook LB. Adult porcine genome-wide DNA methylation patterns support pigs as a biomedical model. BMC Genomics. 2015;16(1):743.

41. Salilew-Wondim D, Fournier E, Hoelker M, Saeed-Zidane M, Tholen E, Looft C, Neuhoff C, Besenfelder U, Havlicek V, Rings F, et al. Genome-wide DNA methylation patterns of bovine blastocysts developed in vivo from embryos completed different stages of development in vitro. PLoS One. 2015;10(11): e0140467.

42. Schroeder DI, Jayashankar K, Douglas KC, Thirkill TL, York D, Dickinson PJ, Williams LE, Samollow PB, Ross PJ, Bannasch DL. Early developmental and evolutionary origins of gene body DNA methylation patterns in mammalian placentas. PLoS Genet. 2015;11(8):e1005442.

43. Zhou Y, Xu L, Bickhart DM, Abdel Hay EH, Schroeder SG, Connor EE, Alexander $L$, Sonstegard TS, Van Tassell CP, Chen H, et al. Reduced 
representation bisulphite sequencing of ten bovine somatic tissues reveals DNA methylation patterns and their impacts on gene expression. BMC Genomics. 2016;17(1):779.

44. Kropp J, Carrillo JA, Namous H, Daniels A, Salih SM, Song J, Khatib H. Male fertility status is associated with DNA methylation signatures in sperm and transcriptomic profiles of bovine preimplantation embryos. BMC Genomics. 2017;18(1):280

45. Duan JE, Jiang ZC, Alqahtani F, Mandoiu I, Dong H, Zheng X, Marjani SL, Chen J, Tian XC. Methylome dynamics of bovine gametes and in vivo early embryos. Front Genet. 2019;10:512.

46. Zhang G-W, Wang L, Chen H, Guan J, Wu Y, Zhao J, Luo Z, Huang W, Zuo F. Promoter hypermethylation of PIWI/piRNA pathway genes associated with diminished pachytene piRNA production in bovine hybrid male sterility. Epigenetics. 2020. https://doi.org/10.1080/15592294.2020.1738026. [Epub ahead of print].

47. Fang L, Zhou Y, Liu S, Jiang J, Bickhart DM, Null DJ, Li B, Schroeder SG, Rosen BD, Cole JB, et al. Comparative analyses of sperm DNA methylomes among human, mouse and cattle provide insights into epigenomic evolution and complex traits. Epigenetics. 2019;14(3):260-76.

48. Andersson L, Archibald AL, Bottema CD, Brauning R, Burgess SC, Burt DW, Casas E, Cheng HH, Clarke L, Couldrey C. Coordinated international action to accelerate genome-to-phenome with FAANG, the Functional Annotation of Animal Genomes project. Genome Biol. 2015;16(1):57.

49. Krueger F, Andrews SR. Bismark: a flexible aligner and methylation caller for bisulfite-Seq applications. Bioinformatics. 2011;27(11):1571-2.

50. Zimin AV, Delcher AL, Florea L, Kelley DR, Schatz MC, Puiu D, Hanrahan F, Pertea G, Van Tassell CP, Sonstegard TS, et al. A whole-genome assembly of the domestic cow Bos taurus. Genome Biol. 2009;10(4):R42.

51. Rosen BD, Bickhart DM, Schnabel RD, Koren S, Elsik CG, Tseng E, Rowan TN, Low WY, Zimin A, Couldrey C, et al. De novo assembly of the cattle reference genome with single-molecule sequencing. GigaScience. 2020;9(3):giaa021.

52. Ziller MJ, Gu H, Müller F, Donaghey J, Tsai LTY, Kohlbacher O, De Jager $\mathrm{PL}$, Rosen ED, Bennett DA, Bernstein BE, et al. Charting a dynamic DNA methylation landscape of the human genome. Nature. 2013;500(7463): 477-81.

53. Hon GC, Rajagopal N, Shen Y, McCleary DF, Yue F, Dang MD, Ren B. Epigenetic memory at embryonic enhancers identified in DNA methylation maps from adult mouse tissues. Nat Genet. 2013:45(10):1198-206.

54. Lister R, Mukamel EA, Nery JR, Urich M, Puddifoot CA, Johnson ND, Lucero J, Huang Y, Dwork AJ, Schultz MD, et al. Global epigenomic reconfiguration during mammalian brain development. Science. 2013;341(6146):1237905.

55. Aguet F, Barbeira AN, Bonazzola R, Brown A, Castel SE, Jo B, Kasela S, KimHellmuth S, Liang Y, Oliva M, et al. The GTEx Consortium atlas of genetic regulatory effects across human tissues. bioRxiv. 2019; 787903. https://doi. org/10.1101/787903.

56. Fang L, Cai W, Liu S, Canela-Xandri O, Gao Y, Jiang J, Rawlik K, Li B, Schroeder SG, Rosen BD et al. Comprehensive analyses of 723 transcriptomes enhance genetic and biological interpretations for complex traits in cattle. Genome Res 2020. https://doi.org/10.1101/gr.250704.119. http://cattlegeneatlas.roslin.ed.ac.uk/. Accessed 19 May 2020.

57. Blake LE, Roux J, Hernando-Herraez I, Banovich NE, Perez RG, Hsiao CJ, Eres I, Cuevas C, Marques-Bonet T, Gilad Y. A comparison of gene expression and DNA methylation patterns across tissues and species. Genome Res. 2020; 30(2):250-62.

58. Bird AP. CpG-rich islands and the function of DNA methylation. Nature. 1986;321(6067):209-13.

59. Lander ES, Linton LM, Birren B, Nusbaum C, Zody MC, Baldwin J, Devon K, Dewar K, Doyle M, FitzHugh W, et al. Initial sequencing and analysis of the human genome. Nature. 2001;409(6822):860-921.

60. Wilkinson MF. Evidence that DNA methylation engenders dynamic gene regulation. Proc Natl Acad Sci. 2015;112(17):E2116.

61. Jones PA. The DNA methylation paradox. Trends Genet. 1999;15(1):34-7.

62. Lorincz MC, Dickerson DR, Schmitt M, Groudine M. Intragenic DNA methylation alters chromatin structure and elongation efficiency in mammalian cells. Nat Struct Mol Biol. 2004;11(11):1068-75.

63. Ball MP, Li JB, Gao Y, Lee J-H, LeProust EM, Park I-H, Xie B, Daley GQ, Church GM. Targeted and genome-scale strategies reveal gene-body methylation signatures in human cells. Nat Biotechnol. 2009;27(4):361-8.

64. Laurent L, Wong E, Li G, Huynh T, Tsirigos A, Ong CT, Low HM, Kin Sung KW, Rigoutsos I, Loring J, et al. Dynamic changes in the human methylome during differentiation. Genome Res. 2010;20(3):320-31.
65. Nothjunge $S$, Nührenberg TG, Grüning BA, Doppler SA, Preissl S, Schwaderer M, Rommel C, Krane M, Hein L, Gilsbach R. DNA methylation signatures follow preformed chromatin compartments in cardiac myocytes. Nat Commun. 2017:8(1):1667.

66. Aravin AA, Hannon GJ. Small RNA silencing pathways in germ and stem cells. Cold Spring Harb Symp Quant Biol. 2008;73:283-90.

67. Kochanek S, Renz D, Doerfler W. DNA methylation in the Alu sequences of diploid and haploid primary human cells. EMBO J. 1993;12(3):1141-51.

68. Chesnokov IN, Schmid CW. Specific Alu binding protein from human sperm chromatin prevents DNA methylation. J Biol Chem. 1995;270(31):18539-42.

69. Eberhart CG, Maines JZ, Wasserman SA. Meiotic cell cycle requirement for a fly homologue of human deleted in Azoospermia. Nature. 1996;381(6585):783-5.

70. Saxena R, Brown LG, Hawkins T, Alagappan RK, Skaletsky H, Reeve MP, Reijo $R$, Rozen S, Dinulos MB, Disteche CM, et al. The DAZ gene cluster on the human $Y$ chromosome arose from an autosomal gene that was transposed, repeatedly amplified and pruned. Nat Genet. 1996;14(3):292-9.

71. Alexander J, Findlay GM, Kircher M, Shendure J. Concurrent genome and epigenome editing by CRISPR-mediated sequence replacement. BMC Biol. 2019:17(1):90.

72. Aran D, Hu Z, Butte AJ. xCell: digitally portraying the tissue cellular heterogeneity landscape. Genome Biol. 2017;18(1):220.

73. Kim-Hellmuth S, Aguet F, Oliva M, Muñoz-Aguirre M, Wucher V, Kasela S, Castel SE, Hamel AR, Viñuela A, Roberts AL, et al. Cell type specific genetic regulation of gene expression across human tissues. bioRxiv. 2019:806117.

74. Villar D, Berthelot C, Aldridge S, Rayner TF, Lukk M, Pignatelli M, Park TJ, Deaville R, Erichsen JT, Jasinska AJ, et al. Enhancer evolution across 20 mammalian species. Cell. 2015;160(3):554-66.

75. Bailey TL, Boden M, Buske FA, Frith M, Grant CE, Clementi L, Ren J, Li WW, Noble WS. MEME Suite: tools for motif discovery and searching. Nucleic Acids Res. 2009;37(suppl_2):W202-8.

76. Zhou Y, Liu S, Hu Y, Fang L, Gao Y, Xia H, et al. Comparative whole genome DNA methylation profiling across cattle tissues reveals global and tissuespecific methylation patterns. Gene Expr Omnibus. 2020. https://www.ncbi. nlm.nih.gov/geo/query/acc.cgi?acc=GSE137943. Accessed 19 May 2020.

77. Zhou Y, Liu S, Hu Y, Fang L, Gao Y, Xia H, et al. Comparative whole genome DNA methylation profiling across cattle tissues reveals global and tissuespecific methylation patterns. Gene Expression Omnibus. 2020. https://www. ncbi.nlm.nih.gov/geo/query/acc.cgi?acc=GSE142727. Accessed 19 May 2020.

78. Zhou Y, Liu S, Hu Y, Fang L, Gao Y, Xia H, et al. Comparative whole genome DNA methylation profiling across cattle tissues reveals global and tissuespecific methylation patterns. Gene Expression Omnibus. 2020. https://www. ncbi.nlm.nih.gov/geo/query/acc.cgi?acc=GSE147087. Accessed 19 May 2020.

\section{Publisher's Note}

Springer Nature remains neutral with regard to jurisdictional claims in published maps and institutional affiliations.

\section{Ready to submit your research? Choose BMC and benefit from:}

- fast, convenient online submission

- thorough peer review by experienced researchers in your field

- rapid publication on acceptance

- support for research data, including large and complex data types

- gold Open Access which fosters wider collaboration and increased citations

- maximum visibility for your research: over $100 \mathrm{M}$ website views per year

At BMC, research is always in progress.

Learn more biomedcentral.com/submissions 